\title{
Fitting B-Spline Curves to Point Clouds by Curvature-Based Squared Distance Minimization
}

\author{
WENPING WANG \\ University of Hong Kong \\ HELMUT POTTMANN \\ Vienna University of Technology \\ and \\ YANG LIU \\ University of Hong Kong
}

Computing a curve to approximate data points is a problem encountered frequently in many applications in computer graphics, computer vision, $\mathrm{CAD} / \mathrm{CAM}$, and image processing. We present a novel and efficient method, called squared distance minimization (SDM), for computing a planar B-spline curve, closed or open, to approximate a target shape defined by a point cloud, that is, a set of unorganized, possibly noisy data points. We show that SDM significantly outperforms other optimization methods used currently in common practice of curve fitting. In SDM, a B-spline curve starts from some properly specified initial shape and converges towards the target shape through iterative quadratic minimization of the fitting error. Our contribution is the introduction of a new fitting error term, called the squared distance (SD) error term, defined by a curvature-based quadratic approximant of squared distances from data points to a fitting curve. The SD error term faithfully measures the geometric distance between a fitting curve and a target shape, thus leading to faster and more stable convergence than the point distance (PD) error term, which is commonly used in computer graphics and CAGD, and the tangent distance (TD) error term, which is often adopted in the computer vision community. To provide a theoretical explanation of the superior performance of SDM, we formulate the B-spline curve fitting problem as a nonlinear least squares problem and conclude that SDM is a quasi-Newton method which employs a curvature-based positive definite approximant to the true Hessian of the objective function. Furthermore, we show that the method based on the TD error term is a Gauss-Newton iteration, which is unstable for target shapes with high curvature variations, whereas optimization based on the PD error term is the alternating method that is known to have linear convergence.

Categories and Subject Descriptors: I.3.5 [Computer Graphics]: Computational Geometry and Object Modeling-Curve, surface, solid, and object representations; J.6 [Computer-Aided Engineering]: —Computer-aided design (CAD); G.1.6 [Numerical Analysis]: Optimization—Least squares methods

General Terms: Algorithms, Theory

Additional Key Words and Phrases: B-spline curve, curve fitting, point cloud, least squares problem, optimization, squared distance, Gauss-Newton method, quasi-Newton method, shape reconstruction, scatter data approximation

The work of W. Wang was partially supported by a CRCG grant from The University of Hong Kong and the national key basic research project of China (2004CB318000). H. Pottmann acknowledges support by the Austrian Science Fund under grant P16002-N05.

Authors' addresses: W. Wang, Department of Computer Science, The University of Hong Kong, Pokfulam Road, Hong Kong, China; email: wenping@cs.hku.hk; H. Pottmann, Institute of Discrete Mathematics and Geometry, Vienna University of Technology, Wien, Austria; email: pottmann@geometrie.tuwien.ac.at; Y. Liu, Department of Computer Science, The University of Hong Kong, Pokfulam Road, Hong Kong, China; email: yliu@cs.hku.hk.

Permission to make digital or hard copies of part or all of this work for personal or classroom use is granted without fee provided that copies are not made or distributed for profit or direct commercial advantage and that copies show this notice on the first page or initial screen of a display along with the full citation. Copyrights for components of this work owned by others than ACM must be honored. Abstracting with credit is permitted. To copy otherwise, to republish, to post on servers, to redistribute to lists, or to use any component of this work in other works requires prior specific permission and/or a fee. Permissions may be requested from Publications Dept., ACM, Inc., 1515 Broadway, New York, NY 10036 USA, fax: +1 (212) 869-0481, or permissions@acm.org. (c) 2006 ACM 0730-0301/06/0400-0214 $\$ 5.00$ 


\section{INTRODUCTION}

We consider the following problem: Given a set of unorganized data points $X_{k}, k=1,2, \ldots, n$, in the plane, compute a planar B-spline curve to approximate the points $X_{k}$. The data points $X_{k}$ are assumed to represent the shape of some unknown planar curve, which can be open or closed, but not self-intersecting; this curve is called a target curve or target shape. We suppose that unorganized data points, often referred to as a point cloud or scattered data points in literature, may have nonuniform distribution with considerable noise; this assumption makes it difficult or impossible to order data points along the target curve. Hence, we assume that such an ordering is not available.

The above problem can be formulated as a nonlinear optimization problem as follows. Consider a Bspline curve $P(t)=\sum_{i=1}^{m} P_{i} B_{i}(t)$ with control points $P_{i}$. We assume throughout that the order and the knots of the B-spline curve are fixed so they are not subject to optimization. This simplifying assumption allows us to give a clear explanation of the basic idea of our new optimization scheme. The very same idea of optimization presented here can also be extended to the more general setting of fitting a NURBS curve with free weights and knots to be optimized by variable linearization and inequality constraints.

Given data points $X_{k}, k=1,2, \ldots, n$, we want to find the control points $P_{i}, i=1,2, \ldots, m$, such that the objective function

$$
f=\frac{1}{2} \sum_{k=1}^{n} d^{2}\left(P(t), X_{k}\right)+\lambda f_{s}
$$

is minimized, where $d\left(P(t), X_{k}\right)=\min _{t}\left\|P(t)-X_{k}\right\|$ is the distance of the data point $X_{k}$ to the curve $P(t), f_{s}$ is a regularization term to ensure a smooth solution curve, and $\lambda$ is a positive constant to modulate the weight of $f_{s}$. Here the distance $d\left(P(t), X_{k}\right)$ is measured orthogonal to the curve $P(t)$ from $X_{k}$. The exceptional case where the shortest distance from $X_{k}$ to an open curve $P(t)$ occurs at an endpoint of $P(t)$ will be discussed separately in Section 5 .

We present a novel method that approximates unorganized data points with a B-spline curve that starts with some properly specified initial curve and converges through iterative optimization towards the target shape of data points. One of our contributions is the introduction of a novel error term defined by a curvature-based quadratic approximant of squared distances from data points to the fitting curve. For brevity, this new error term is called the squared distance error term ( $S D$ error term), and the resulting iterative minimization scheme will be referred to as squared distance minimization (SDM). Because the SD error term faithfully measures the geometric distance between data points and the fitting curve, SDM converges fast and stably in comparison with other commonly used error terms as will be discussed shortly.

The remainder of the article is organized as follows. We first review related previous work. Then we introduce the SD error term, outline our SDM method, and use some test examples to show the superior performance of SDM in comparison with two other commonly used methods, point distance minimization (PDM) and tangent distance minimization (TDM). We will also discuss the B-spline curve fitting problem from the viewpoint of optimization to provide insights into the superior performance of SDM. We will show that SDM is, in fact, a quasi-Newton method which employs a carefully chosen positive definite approximant to the true Hessian of the objective function. We will also show that TDM is a variant of the Gauss-Newton method. Furthermore, PDM is, in fact, the alternating method for solving a separable problem and is known to have only linear convergence [Ruhe and Wedin 1980; Bjorck 1996; Speer et al. 1998]. This systematic study of the relationship between these geometrically motivated curve fitting methods and standard optimization techniques is another contribution of our work. 


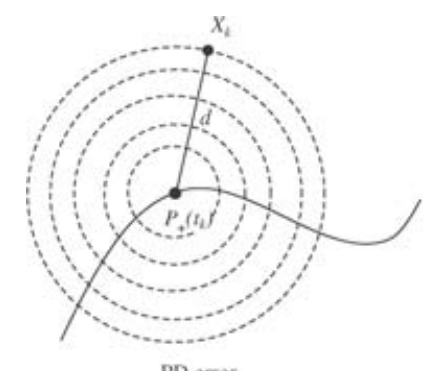

Fig. 1. Iso-values curves of the point distance (PD) error term.

\section{RELATED WORK}

\subsection{Spline Curve Fitting Techniques}

Fitting a curve to a set of data points is a fundamental problem in graphics (e.g., Pavlidis [1983]; Plass and Stone [1983]; Pratt [1985]; Walton and Xu [1991]; Goshtasby [2000]) and many other application areas. Instead of attempting a comprehensive review, we will only discuss some main results in the literature to provide a background for our work.

Let $X_{k} \in R^{2}, k=1,2, \ldots, n$, be unorganized data points representing a target shape which is to be approximated by a closed or open planar B-spline curve $P(t)=\sum_{i=1}^{m} B_{i}(t) P_{i}$, where the $B_{i}(t)$ are the Bspline basis functions of a fixed order and knots, and the $P_{i}$ are the control points. Since $f$ in Equation (1) is a nonlinear objective function, iterative minimization comes as a natural approach. Suppose that we have a specific B-spline curve $P_{c}(t)=\sum_{i=1}^{m} B_{i}(t) P_{c, i}$ with control points $\mathcal{P}_{c}=\left(P_{c, 1}, P_{c, 2}, \ldots, P_{c, m}\right)$, which can be an initial fitting curve or the current fitting generated from the last iteration. Let $\mathcal{D}=$ $\left(D_{1}, D_{2}, . ., D_{m}\right)$ be the variable updates to $\mathcal{P}_{c}$ to give the new control points $\mathcal{P}_{+}=\mathcal{P}_{c}+\mathcal{D}$. Let $P_{+}(t)=$ $\sum_{i=1}^{m} B_{i}(t)\left(P_{c, i}+D_{i}\right)$ denote the B-spline curve with updated control points $\mathcal{P}_{+}$.

Many existing B-spline curve fitting methods invoke a data parameterization procedure to assign a parameter value $t_{k}$ to each data point $X_{k}$. In some methods dealing with ordered data points, the chord length method or the centripetal method [Lee 1989; Hoschek and Lasser 1993; Farin 1997] are used for data parameterization. Then, with the fixed $t_{k}$, the function

$$
\hat{f}=\frac{1}{2} \sum_{k}\left\|P_{+}\left(t_{k}\right)-X_{k}\right\|^{2}+\lambda f_{s}
$$

which is a local quadratic model of $f$ in (1), is minimized by solving a linear system of equations to yield updated control points $\mathcal{P}_{+}$, and hence the updated fitting curve $P_{+}(t)$.

A commonly used data parameterization method is to choose $t_{k}$ such that $P_{c}\left(t_{k}\right)$ is the closest point from the current fitting curve to the data point $X_{k} ;\left(P_{c}\left(t_{k}\right)\right.$ is also called the foot point of $X_{k}$ on the curve $P_{c}(t)$ ). Then an iterative method can be developed by interleaving this step of foot point computation with the minimization of the local quadratic model $\hat{f}$ in Equation (2) to compute the updated control points $\mathcal{P}_{+}$. Note that the error term $\left\|P_{+}\left(t_{k}\right)-X_{k}\right\|^{2}$ in Equation (2) measures the squared distance between the data point $X_{k}$ and a particular point $P_{+}\left(t_{k}\right)$ on the fitting curve to be determined; therefore, we will call this error term the point distance error term (PD error term), and denote it by

$$
e_{P D, k}=\left\|P_{+}\left(t_{k}\right)-X_{k}\right\|^{2} \text {. }
$$

(The PD error term is illustrated in Figure 1.) This minimization scheme will be called the point distance minimization or PDM for short. Note that, since the ordering of data points is not required for data parameterization via foot point projection, PDM is applicable to fitting a curve to a point cloud. 


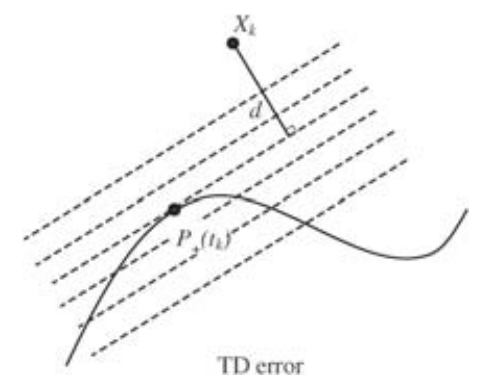

Fig. 2. Iso-values curves of the tangent distance (TD) error term.

Hoschek [1988] proposes an iterative scheme, called intrinsic parameterization, which also uses the PD error term but performs parameter correction using a formula that is a first-order approximation to exact foot point computation. Bercovier and Jacob [1994] prove that the intrinsic parameterization method is equivalent to Uzawa's method for solving a constrained minimization problem, but they do not establish the convergence rate of the intrinsic parameterization method or that of PDM. Higher order approximation or accurate computation of foot points for data parameterization are discussed in Hoschek and Lasser [1993]; Saux and Daniel [2003]; Hu and Wallner [2005].

PDM, or its simple variants, are the most commonly applied methods for curve fitting in computer graphics and CAD [Plass and Stone 1983; Hoschek 1988; Goshtasby 2000; Saux and Daniel 2003]. The same idea of PDM is also widely used for surface fitting [Hoppe et al. 1994; Forsey and Bartels 1995; Hoppe 1996; Ma and Kruth 1995; Haber et al. 2001; Wang and Phillips 2002; Djebali et al. 2002; Greiner et al. 2002; Weiss et al. 2002; Maekawa and Ko 2002; Taubin 2002] with B-spline surfaces as well as other types of surfaces. The popularity of PDM might be explained by its simplicity-the error term $e_{P D, k}$ in Equation (3) is derived by simply substituting $t_{k}$ in the squared distance $d^{2}\left(P(t), X_{k}\right)$ in the original objective function $f$ in Equation (1). However, considering that $P\left(t_{k}\right)$ is a variable point depending on the variable control points, $e_{P D, k}$ is a rather poor approximation to $d^{2}\left(P(t), X_{k}\right)$, thus causing slow convergence. As a matter of fact, the present article is just about how to use a better approximation of $d^{2}\left(P(t), X_{k}\right)$ to devise a more efficient optimization scheme.

Another error term, often used in the computer vision community (e.g., Blake and Isard [1998]), is defined by

$$
e_{T D, k}=\left[\left(P_{+}\left(t_{k}\right)-X_{k}\right)^{T} N_{k}\right]^{2},
$$

where $N_{k}$ is a unit normal vector of the current fitting curve $P_{c}(t)$ at the point $P_{c}\left(t_{k}\right)$. We will call $e_{T D, k}$ the tangent distance error term (TD error term) since $e_{T D, k}$ gives the squared distance from $X_{k}$ to the tangent of $P_{c}(t)$ at the foot point $P_{c}\left(t_{k}\right)$ when $P_{+}(t)$ is $P_{c}(t)$. The TD error term is illustrated in Figure 2.

The TD error term $e_{T D, k}=\left[\left(P_{+}\left(t_{k}\right)-X_{k}\right)^{T} N_{k}\right]^{2}$ can also be combined with data parametrization via foot point projection to yield a B-spline curve fitting method as used in Blake and Isard [1998] for boundary extraction in motion tracking. We will call this method tangent distance minimization (TDM). TDM minimizes in each iteration the function

$$
f_{T D}=\frac{1}{2} \sum_{k} e_{T D, k}+\lambda f_{s}
$$

Treating the control points $\mathcal{P}_{+}$as variables to be optimized, the TD error term measures the squared distance from the point $X_{k}$ to a moving straight line $L_{k}$ that has the fixed normal vector $N_{k}$ and passes through the moving point $P_{+}\left(t_{k}\right)$, (see Figure 3). The TD error $e_{T D, k}=\left[\left(P_{+}\left(t_{k}\right)-X_{k}\right) \cdot N_{k}\right]^{2}$ becomes zero if the point $X_{k}$ is contained in the line $L_{k}$. On the other hand, the line $L_{k}$ is a relatively 


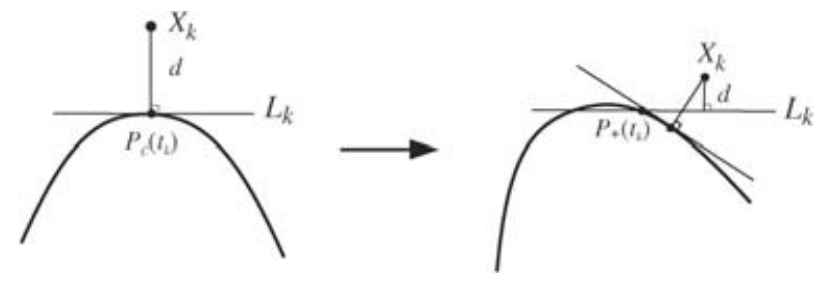

Fig. 3. In a neighborhood of a high curvature point, the true approximation error can be rather big even when the TD error $d$ is small.

poor approximation to the curve $P_{+}(t)$ in a neighborhood of a high-curvature point $P_{+}\left(t_{k}\right)$ or if $X_{k}$ is far from $P_{+}\left(t_{k}\right)$. Hence, in these cases, the point $X_{k}$ may still be poorly approximated by the curve $P_{+}(t)$ even if $X_{k}$ is nearly on $L_{k}$, that is, when the TD error $\left[\left(P_{+}\left(t_{k}\right)-X_{k}\right) \cdot N_{k}\right]^{2}$ is nearly zero (see Figure 3).

This disparity between approximation quality and error measurement is the cause of the instability of TDM near a high-curvature part of the target shape as will be illustrated later with test examples. This unstable behavior of TDM is, in fact, the consequence of using an inappropriately large step size to solve a nonlinear optimization problem; indeed, we will show that TDM uses Gauss-Newton iteration for solving a nonlinear least-squares problem and its excessively large step size is due to omission of important curvature related parts in the true Hessian.

Now let us consider the geometric interpretations of the PD error term and the TD error term. Since $P_{c}\left(t_{k}\right)$ is the fixed foot point on the current fitting curve $P_{c}(t)$ of $X_{k}$, if $P_{+}(t)$ is the same as $P_{c}(t)$, then both $e_{P D, k}$ and $e_{T D, k}$ give the same value of $d^{2}\left(P_{c}\left(t_{k}\right), X_{k}\right)$. However, for optimization purpose, we need to regard $d^{2}\left(P_{+}\left(t_{k}\right), X_{k}\right)$ as a function of variable control points $\mathcal{P}_{+}$, and, in this sense, $e_{P D, k}$ and $e_{T D, k}$ give very different approximations to $d^{2}\left(P_{+}\left(t_{k}\right), X_{k}\right)$.

If treating $X_{k}$ as a free point for any constant $c>0$, the iso-value curve $e_{P D, k} \equiv\left\|P_{+}\left(t_{k}\right)-X_{k}\right\|^{2}=c$ of the PD error term is a circle (see Figure 1), and the iso-value curve $e_{T D, k} \equiv\left[\left(P_{+}\left(t_{k}\right)-X_{k}\right) \cdot N_{k}\right]^{2}=c$ of the TD error term is a pair of parallel lines which can be regarded as a degenerate ellipse (see Figure 2). Since PDM has relatively slow convergence and TDM tends to have fast but unstable convergence, one may speculate whether or not a new error term with ellipse-shaped iso-value curves can be devised to yield a more balanced performance between efficiency and stability. We will see that such an error term is naturally provided by a curvature-based quadratic approximation to the squared distance function.

\subsection{Second Order Approximation to Squared Distance Function}

Given a curve $\mathcal{C}$ in $E^{2}$, one can define the squared distance function that assigns to each point $X$ in $E^{2}$ the squared distance from $X$ to $\mathcal{C}$. The second order approximation to this distance function is given in Ambrosio and Montegazza [1998]. This approximation is then studied in detail and applied to solving a number of shape fitting problems in Pottmann et al. [2002]; Pottmann and Hofer [2003]. We review this work briefly.

Let $O$ be the closest point on a twice differentiable curve $\mathcal{C}$ to a fixed point $X_{0}$ (see Figure 4). Consider the local Frenet frame of $\mathcal{C}$ with its origin at $O$ and its two coordinate axes parallel to the tangent vector and the normal vector of the curve $\mathcal{C}$ at $O$. Let $\rho>0$ be the curvature radius of $\mathcal{C}$ at $O$. We use an orientation of the curve normal such that $K=(0, \rho)^{T}$ is the curvature center of $\mathcal{C}$ at $O$. Let $d$ be the signed distance from $X_{0}$ to $O$, that is, $|d|=\left\|X_{0}-O\right\|$ with $d<0$ if $X_{0}$ and $K$ are on opposite sides of the curve $\mathcal{C}$, and $d>0$ if $X_{0}$ and $K$ are on the same side of $\mathcal{C}$. We note that there is always $d<\rho$ when $d>0$, for otherwise $O$ cannot be the closest point on the curve $\mathcal{C}$ to $X_{0}$. 


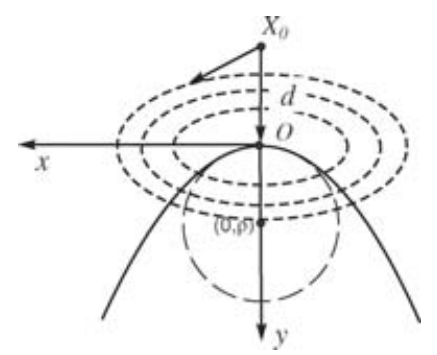

Fig. 4. A second order approximant of the squared distance function to the curve $C$ at $X_{0}$.

Consider a point $X=(x, y)^{T}$ in a neighborhood of $X_{0}$. The second order approximant of the squared distance from $X$ to the curve $\mathcal{C}$ is

$$
g(x, y)=\frac{d}{d-\rho} x^{2}+y^{2}
$$

[Ambrosio and Montegazza 1998; Pottmann and Hofer 2003]. Geometrically, the conic section $g(x, y)=$ $d^{2}$ has second order contact with the offset curve of the target curve $\mathcal{C}$ that passes through $X_{0}$.

Since $g(x, y)$ in Equation (6) is indefinite when $0<d<\rho$, the unified expression

$$
\hat{g}(x, y)=\frac{|d|}{|d|+\rho} x^{2}+y^{2}
$$

is used in Pottmann et al. [2002] as a positive semidefinite quadratic error term for solving geometric optimization problems. Note that, with this modification to Equation (6) in the case of $0 \leq d<\rho$, $\hat{g}(x, y)$ becomes a first order approximation to the squared distance function to $\mathcal{C}$ in a neighborhood of $X_{0}$. An alternative to this modification is to replace the first coefficient $d /(d-\rho)$ by $\max \{0, d /(d-\rho)\}$, thus also making the distance measurement positive semidefinite in all cases.

This approximation to the squared distance of a smooth target curve is used in Pottmann et al. [2002] for fitting a B-spline curve to the target curve as follows. Given a target curve to be approximated and the current B-spline fitting curve $P_{c}(t)$ with control points $\mathcal{P}_{c}=\left\{P_{c, i}\right\}$, a set of densely distributed points $S_{k}$, called sensor points, are first sampled on $P_{c}(t)$. Then the approximate squared distance $f_{k}\left(S_{k}\right)$ defined by Equation (7) from each sensor point $S_{k}$ to the fixed target curve is computed. Let $\mathcal{P}_{+}=\mathcal{P}_{c}+\mathcal{D}$ denote the updated control points of the B-spline fitting curve, where $\mathcal{D}$ are the incremental updates to the current control points $\mathcal{P}_{c}$. The error term associated with each sensor point is defined as

$$
\hat{e}_{k}=\hat{g}\left(S_{k}\left(\mathcal{P}_{+}\right)\right)
$$

which is quadratic in the control points $\mathcal{P}_{+}$, since each $S_{k}$ is a linear combination of the control points. Then the local error function $f=\frac{1}{2} \sum_{k} \hat{e}_{k}+\lambda f_{s}$, where $f_{s}$ is a regularization term that is quadratic in $\mathcal{P}_{+}$, is minimized to find the updated control points $\mathcal{P}_{+}$by solving a linear system of equations. This minimization step is iterated to make the fitting curve $P(t)$ move towards the target curve.

The superior efficiency of the previous curve fitting scheme comes from the fact that the error function $\hat{e}_{k}$ in Equation (8) is an accurate approximation to the true squared distance function from $S_{k}$ to the target curve $\mathcal{C}$ in terms of the incremental updates $\mathcal{D}$. However, this method assumes that the target shape is a smooth curve whose tangent and curvature information can easily be evaluated or estimated accurately, thus it is not applicable to a target point defined by a point cloud because of the difficulty in computing accurate tangent and curvature from noisy or sparsely distributed data points. 


\section{FITTING A B-SPLINE CURVE TO A POINT CLOUD USING SDM}

In this section, we introduce a new SD error term for fitting a B-spline curve to a point cloud. We emphasize that this new SD error term is defined by a quadratic approximation to the squared distance function of the B-spline fitting curve, rather than that of the fixed target shape. In other words, we measure the fitting error as defined in Equation (1), namely, orthogonal to the fitting curve, in contrast to the method in Pottmann et al. [2002] (or see Section 2.2) in which errors are measured orthogonal to the fixed target curve and therefore also a different objective function is minimized.

\subsection{A New Quadratic Approximation to the Squared Distance}

Given the current B-spline fitting curve $P_{c}(t)=\sum_{i=1}^{m} B_{i}(t) P_{c, i}$, let $P_{+}(t)$ denote the fitting curve with updated control points $\mathcal{P}_{+}=\mathcal{P}_{c}+\mathcal{D}$, where $\mathcal{P}_{c}=\left\{P_{c, i}\right\}$ and $\mathcal{D}$ are incremental updates to $\mathcal{P}_{c}$. Suppose that $P_{c}\left(t_{k}\right)$ is the foot point of the data point $X_{k}$ on $P_{c}(t)$. Let $T_{k}$ and $N_{k}$ be the unit tangent vector and the unit normal vector of the current fitting curve $P_{c}(t)$ at the foot point $P_{c}\left(t_{k}\right), \rho>0$ is the curvature radius of $P_{c}(t)$ at $P_{c}\left(t_{k}\right)$, and $|d|=\left\|P_{c}\left(t_{k}\right)-X_{k}\right\|$ with the same convention on the sign of $d$ as made in Section 2.2. When the control points $\mathcal{P}_{+}$change, with the same parameter $t_{k}$, the foot point $P_{c}\left(t_{k}\right)$ becomes a variable point $P_{+}\left(t_{k}\right)$, and the unit tangent vector $\tilde{T}_{k}$, the unit normal vector $\tilde{N}_{k}$, and curvature radius $\tilde{\rho}$ of the curve $P_{+}(t)$ at the point $P_{+}\left(t_{k}\right)$, all vary with $\mathcal{P}_{+}$.

To obtain a quadratic approximation to the squared distance from $X_{k}$ to the curve $P_{+}(t)$, we assume that $\tilde{T}_{k}, \tilde{N}_{k}$, and $\tilde{\rho}$ are fixed to be $T_{k}, N_{k}$, and $\rho$, that is, they do not vary with $\mathcal{P}_{+}$. This assumption implies that, locally at the point $P_{c}\left(t_{k}\right)$, we will only consider a differential translation of the curve $P_{c}(t)$ into the curve $P_{+}(t)$. Since this translation is relative to the data point $X_{k}$, we may view, in a relative sense, that $P_{+}(t)$ is a fixed curve and $X_{k}$ undergoes a translation. Therefore, we may use Equation (6) to approximate the squared distance from $X_{k}$ to the curve $P_{+}(t)$, expressed in the global coordinate system as

$$
h_{k}(\mathcal{D})=\frac{d}{d-\rho}\left[\left(P_{+}\left(t_{k}\right)-X_{k}\right)^{T} T_{k}\right]^{2}+\left[\left(P_{+}\left(t_{k}\right)-X_{k}\right)^{T} N_{k}\right]^{2} .
$$

Note that, $h_{k}(\mathcal{D})$ may take a negative value when $0<d<\rho$. In order to obtain a positive semidefinite error metric based on $h_{k}(\mathcal{D})$, we define the new error term as

$$
e_{S D, k}(\mathcal{D})= \begin{cases}\frac{d}{d-\rho}\left[\left(P_{+}\left(t_{k}\right)-X_{k}\right)^{T} T_{k}\right]^{2}+\left[\left(P_{+}\left(t_{k}\right)-X_{k}\right)^{T} N_{k}\right]^{2}, & \text { if } d<0, \\ {\left[\left(P_{+}\left(t_{k}\right)-X_{k}\right)^{T} N_{k}\right]^{2},} & \text { if } 0 \leq d<\rho,\end{cases}
$$

Clearly, $e_{S D, k}(\mathcal{D})$ is a positive semidefinite quadratic function of $\mathcal{D}$ in all cases. Since $e_{S D, k}(\mathcal{D})$ is derived from a direct attempt to accurately approximate the squared distance function, we will call $e_{S D, k}(\mathcal{D})$ the squared distance error term or $S D$ error term for short. We stress that, due to the simplifications we have made, $e_{S D, k}(\mathcal{D})$ is, in general, no longer a second order approximation to the squared distance function, but rather a first order approximation that is more accurate than the PD error term or the TD error term.

When $d<0$, the level-set curve of $e_{S D, k}(\mathcal{D})=c$ is an ellipse centered at the point $P_{+}\left(t_{k}\right)$ if the $X_{k}$ is treated as a variable point. When the control points $\mathcal{P}_{+}$change, the ellipse is translated by keeping its center at $P_{+}\left(t_{k}\right)$ but with its shape, size, and orientation remaining unchanged (see Figure 5). The SD error term $e_{S D, k}$ becomes the TD error term $e_{T D, k}$ when $0 \leq d<\rho$, that is, when (i) the data point $X_{k}$ is sufficiently near $P_{c}\left(t_{k}\right)$ relative to the magnitude of $\rho$; and (ii) $X_{k}$ is on the convex side of the curve $P_{c}(t)$, that is, $X_{k}$ and the curvature center $K$ are on the same side of the curve $P_{c}(t)$. The use of the TD error term here will not cause instability since, in this case, the tangent line is a relatively good approximation to the curve $P_{c}(t)$ in a neighborhood of $P_{c}\left(t_{k}\right)$. 


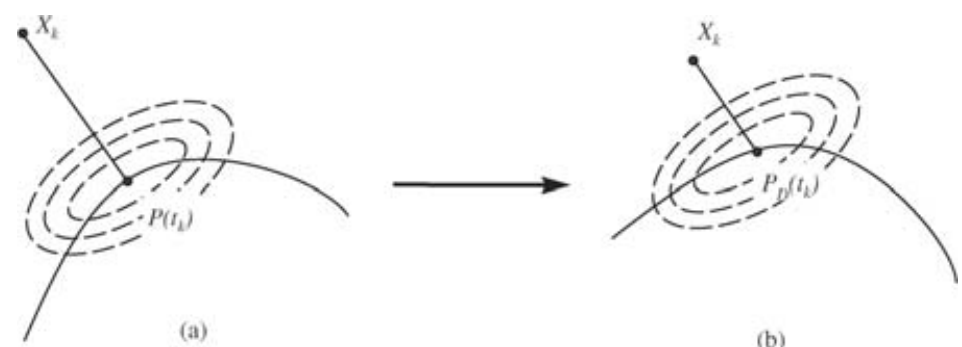

Fig. 5. The SD error term $e_{S D, k}(\mathcal{D})$ defined by Equation (10) is shown via its iso-value curves in the case of $d<0$. (a) Before updating the control points $\mathcal{P}$. (b) The translation of $e_{S D, k}(\mathcal{D})$ after updating $\mathcal{P}$.

Iterative minimization of the squared distance using the SD error term Equation (10), interleaved with foot point computation for data parameterization, will be called squared distance minimization $(S D M)$. Although the tangent and normal vectors $T_{k}$ and $N_{k}$, as well as $\rho$, are kept fixed during an iteration, they are updated at the beginning of each iteration to reflect the continual change of the shape of the fitting curve.

The derivation of the SD error term presented is based on a geometric argument. Using a second order Taylor expansion, we will reveal the relationship between this SD error term and the Newton iteration later in Section 6.3, thus providing another derivation of the SD error term.

\subsection{Main Steps of SDM}

The main steps of the SDM method are as follows.

(1) Specify a proper initial shape of a B-spline fitting curve.

(2) Compute SD error terms for all data points to obtain a local quadratic approximation $f_{S D}$ of the objective function $f$, defined by

$$
f_{S D}=\frac{1}{2} \sum_{k} e_{S D, k}+\lambda f_{s}
$$

(3) Solve a linear system of equations to minimize $f_{S D}$ to obtain an updated B-spline fitting curve.

(4) Repeat steps 2 and 3 until convergence, that is, until a prespecified error threshold is satisfied or the incremental change of the control points falls below a preset threshold.

\section{EXPERIMENTS AND COMPARISON}

In this section, we use some test examples to compare SDM with PDM and TDM for fitting a closed B-spline curve to unorganized data points in the plane. The quadratic function to be optimized in each iteration has the form

$$
f=\frac{1}{2} \sum_{k=1}^{n} e_{k}+\alpha F_{1}+\beta F_{2}
$$

where $e_{k}$ is a particular error term (PD, TD, or SD) for the data point $X_{k}, F_{1}$, and $F_{2}$ are energy terms defined as

$$
F_{1}=\int\left\|P^{\prime}(t)\right\|^{2} \mathrm{~d} t, \quad F_{2}=\int\left\|P^{\prime \prime}(t)\right\|^{2} \mathrm{~d} t
$$

and $\alpha, \beta \geq 0$ are constants. In our implementation, $F_{1}$ and $F_{2}$ are integrated explicitly without numerical approximation. 


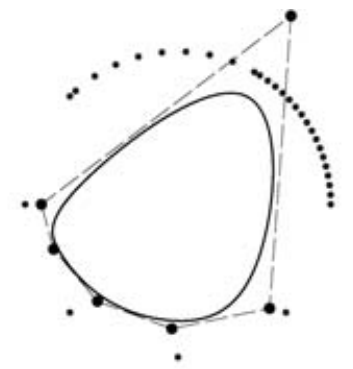

(a) Data points on a circle and an initial B-spline curve.

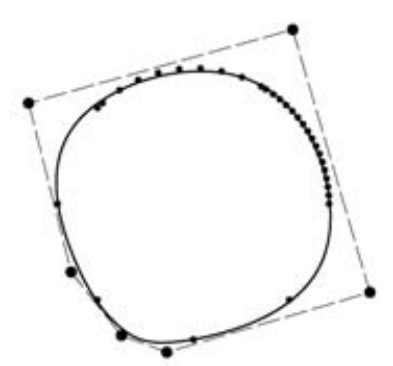

(b) The fitting curve generated by PDM in 10 iterations.

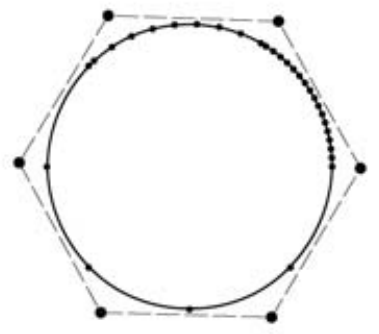

(c) The fitting curve generated by TDM in 10 iterations.

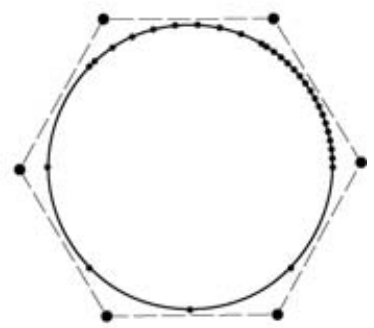

(d) The fitting curve generated by SDM in 10 iterations.

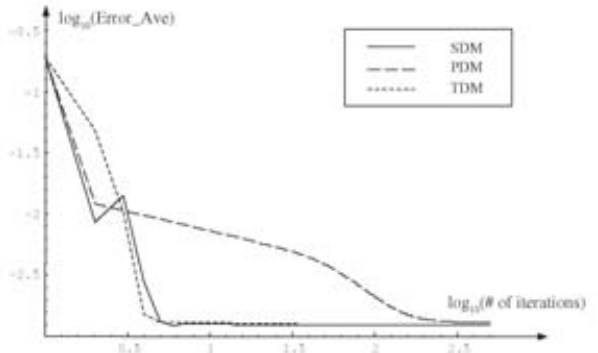

(e) The average error versus the number of iterations of the three methods.

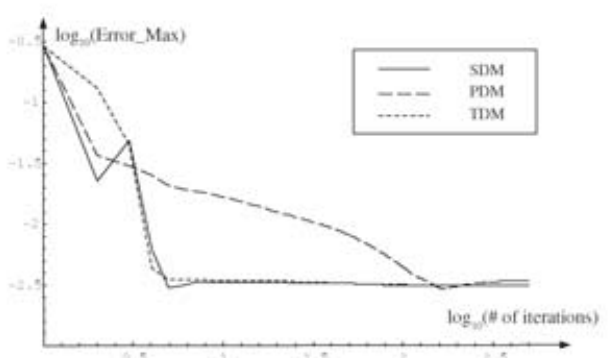

(f) The maximum error versus the number of iterations of the three methods.

Fig. 6. (Example 1) Comparison of the three methods on a set of 32 sparse data points on a circle. In this figure, log scale (base 10 ) is used for the iteration axis in (e) and (f) in order to distinguish the error curves of TDM and SDM.

For a fixed B-spline fitting curve $P(t)$, the Euclidean distance from data point $X_{k}$ to $P(t)$ is denoted by $d_{k}=\left\|P\left(t_{k}\right)-X_{k}\right\|$, where $P\left(t_{k}\right)$ is the foot point of $X_{k}$ on $P(t)$. Then, for evaluating the approximation error, we define the average error which is the root mean square error as

$$
\text { Error Ave }=\left[\frac{1}{n} \sum_{k=1}^{n} d_{k}^{2}\right]^{1 / 2},
$$

and the maximum error as

$$
\text { Error } \operatorname{Max}=\max _{k=1}^{n}\left\{d_{k}\right\} .
$$

In the following, we present the results of applying the three methods-PDM, TDM, and SDM-to fitting a cubic B-spline curve with uniform knots to several sets of unorganized data points. The same values of energy coefficients $\alpha=0$ and $\beta=0.001$ are used for all the examples in this section unless specified otherwise.

Example 1. Nonuniform data points on a circle (see Figure 6). TDM and SDM converge with roughly the same speed, and both converge much faster than PDM does as shown in Figures 6(e) and (f). PDM takes about 100 iterations to reach the same small error values produced by SDM in fewer than 10 iterations.

Example 2. (See Figure 7.) For this set of data points, SDM again converges much faster than PDM does, while TDM is trapped in a local minimum, producing a curve with self-intersection. To visualize 


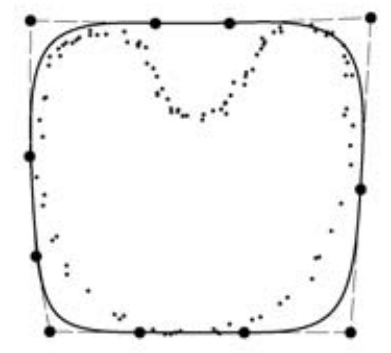

(a) Unorganized data points on a " $\mathrm{C}$ " shape and an initial B-spline curve.

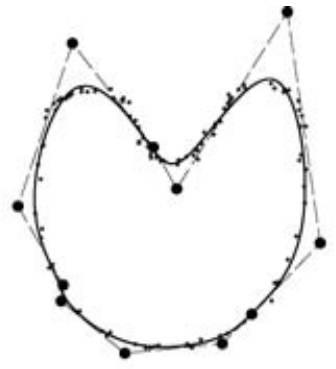

(b) The fitting curve generated by PDM in 20 iterations.

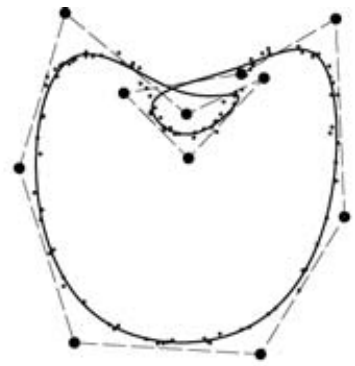

(c) The fitting curve generated by TDM in 20 iterations.

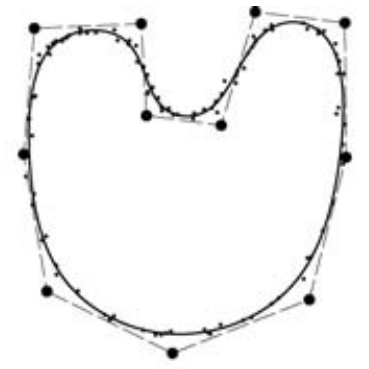

(d) The fitting curve generated by SDM in 20 iterations.

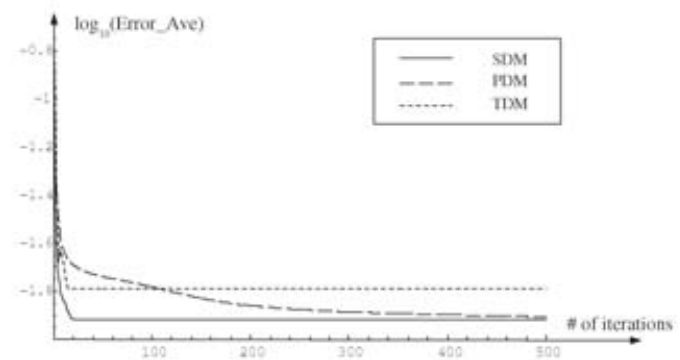

(e) The average error versus the number of iterations of the three methods.

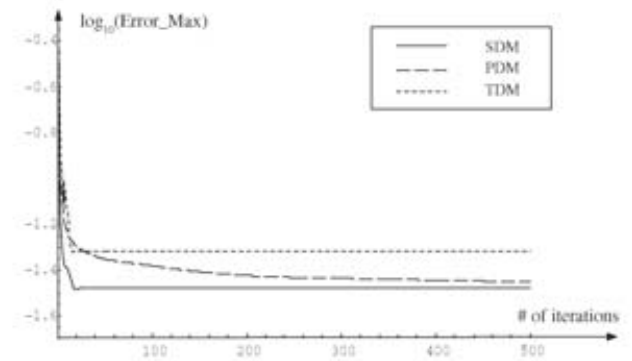

(f) The maximum error versus the number of iterations of the three methods.

Fig. 7. (Example 2) Comparison of the three methods on a set of 102 data points. SDM converges faster than PDM does. TDM is trapped in a local minimum with self-intersection in the fitting curve.

the evolution of an iterative optimization process (PDM or SDM), we place the fitting curves generated in successive iterations at successive heights to form an evolution surface (see Figure 8). Data points or a subset of them are displayed at the top of an evolution surface. A striped texture is used to depict the trajectories of points of fixed parameter values on the fitting curve. The trajectories of evolving control points are shown by white curves in space. Log scale (base 10) is used for the height axis in these figures to accommodate for the large number of iterations needed by PDM. For size reference, a base square of size $2.2 \times 2.2$ is shown along with these evolution surfaces.

The evolution surfaces generated by PDM and SDM (Figure 8), viewed from two different directions, show that PDM experiences a slow convergence process, while SDM converges quickly with conspicuous tangent flow of the control points in the first 10 iterations.

Example 3. (See Figure 9.) This set of data points is extremely noisy. After 50 iterations, SDM has already produced an acceptable result, while PDM converges slowly and TDM becomes unstable. Here strong tangential flow of control points is observed in SDM to move some control points at the bottom of the initial curve to the top in (d).

Example 4. (See Figure 10.) The difficulty with this test lies in the corner points of the target shape and the highly nonuniform distribution of the control points of the initial B-spline curve. After 20 iterations, PDM gets trapped in an unacceptable local minimum and TDM becomes divergent, while SDM converges successfully. Again SDM exhibits strong tangential flow responsible for redistributing control points initially clustered at one side on the initial curve over the target shape to well approximate the four corner points. 


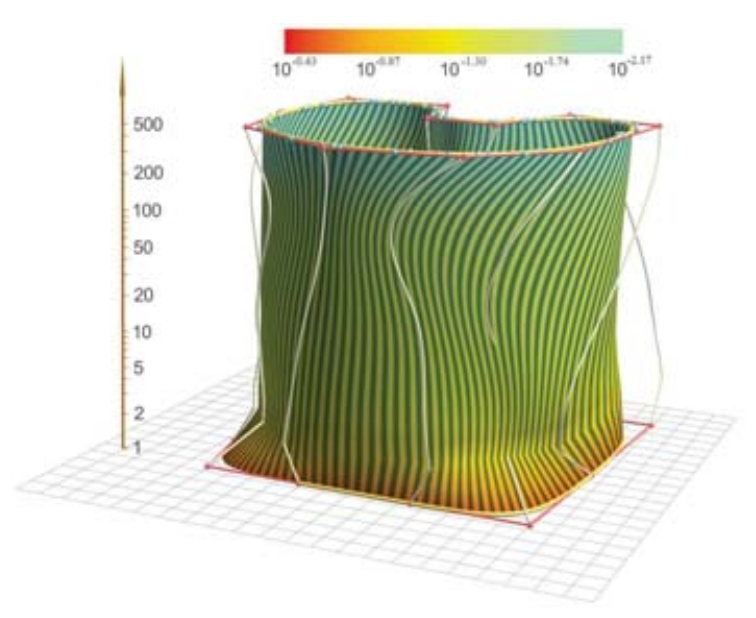

(a) PDM

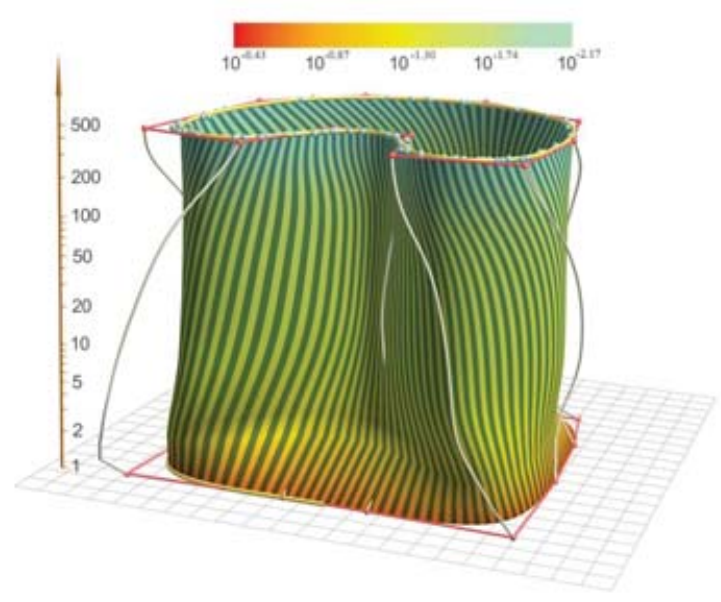

(c) PDM

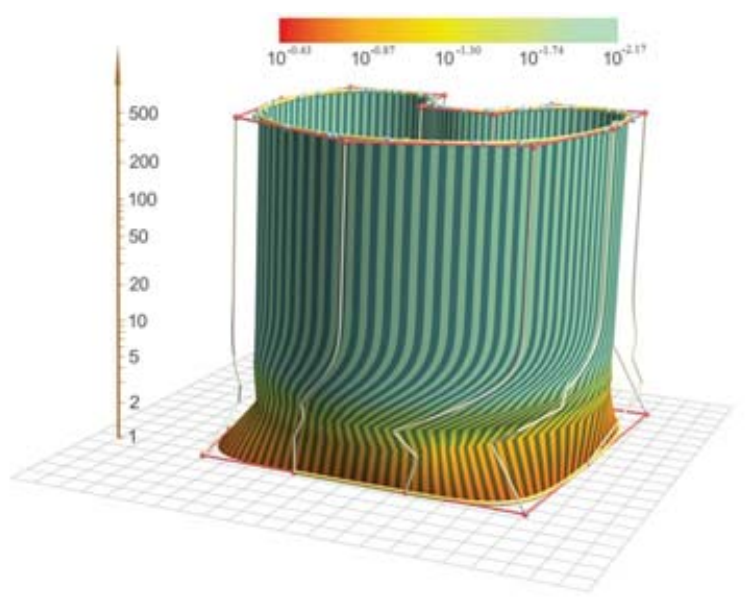

(b) SDM

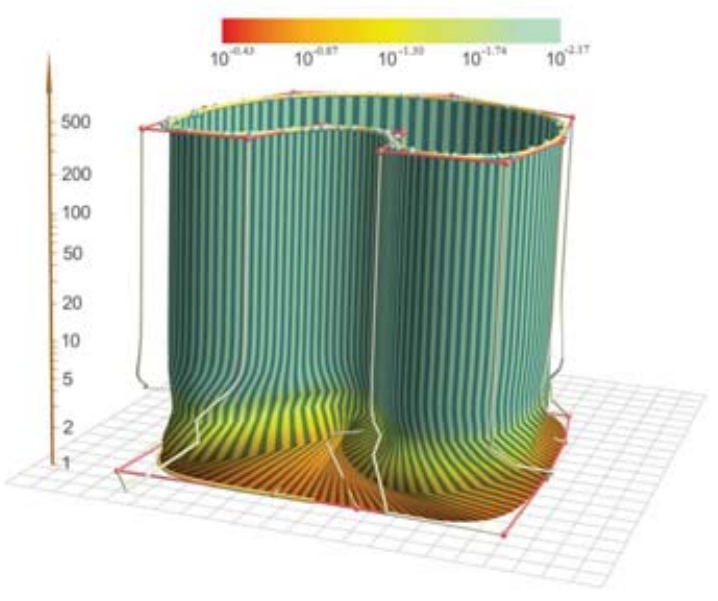

(d) SDM

Fig. 8. The evolution surfaces of PDM and SDM for the data in Example 2 (Figure 7) viewed from two different angles. (a) The evolution surface of PDM; (b) The evolution surface of SDM with the same view angle as in (a); (c) The evolution surface of PDM from another view angle; (d) The evolution surface of SDM with the same view angle as in (c).

The four examples presented are from numerous examples we have experimented with. The following observations can be made from our experiments.

1) PDM exhibits the slowest convergence among the three methods and is often trapped at a poor local minimum. Our experiments confirm the theoretical conclusion that PDM has, in general, only linear convergence. This is further explained in Section 6.

2) TDM demonstrates fast convergence when the target shape is not so noisy (i.e., representing a small residue problem) and the initial fitting curve is relatively near the target shape (i.e., $|d| \leq \rho$ ), but often becomes unstable or even develops self-intersection in a high curvature region of the target shape or if the initial fitting curve is relatively far from the target shape. Increasing the value of the 


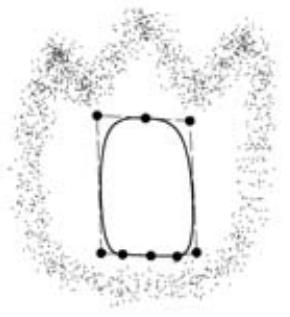

(a) A closed target shape and an initial B-spline curve.

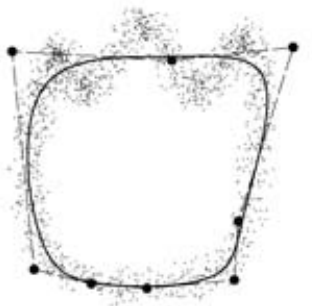

(b) The fitting curve generated by PDM in 50 iterations.

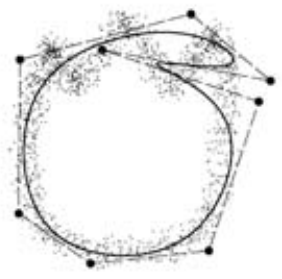

(c) The fitting curve generated by TDM in 50 iterations.

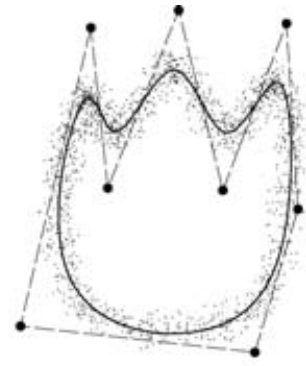

(d) The fitting curve generated by SDM in 50 iterations.

Fig. 9. (Example 3) Comparison of the three methods for fitting an extremely noisy data set of 1,630 points. After 50 iterations, SDM generates a satisfactory fitting curve (d), but TDM becomes unstable (c), and PDM is still improving at a slow rate (b); PDM needs about 400 iterations to produce a fitting curve similar to the one by SDM shown in (d).

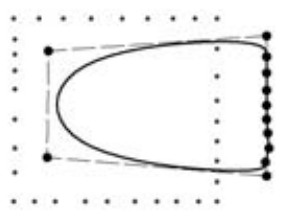

(a) A square-shaped target shape and an initial B-spline curve.

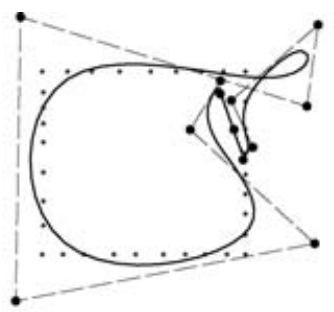

(b) The fitting curve generated by PDM in 20 iterations.

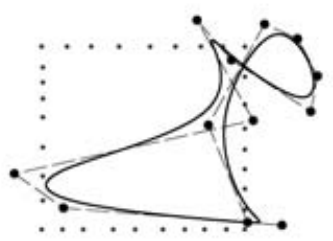

(c) The fitting curve generated by TDM in 20 iterations.

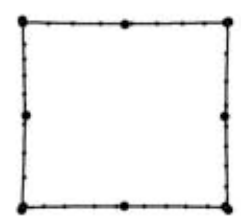

(d) The fitting curve generated by SDM in 20 iterations.

Fig. 10. (Example 4) Comparison of the three methods for approximating a square-shaped target consisting of 33 data points. PDM is trapped in a poor local minimum (b), TDM eventually becomes divergent (c), and SDM converges successfully in 20 iterations $(d)$.

energy coefficient $\beta$ in Equation (11) usually improve the stability of TDM as well as the fairness of the fitting curve but often at the expense of a larger fitting error.

3) SDM exhibits much faster convergence than PDM does. The convergence of SDM is about as fast as that of TDM. Moreover, SDM is more stable than TDM since TDM often does not converge for target shapes with sharp features. This is mainly due to the fact that TDM is a Gauss-Newton method without step size control. We will discuss this in more detail in Section 6.

4) The iso-value curves of the SD error term are ellipses aligned with the tangent at a point of the fitting curve. Therefore, at a low-curvature region of a B-spline fitting curve, the control points of the fitting curve, as well as points on the fitting curve, can flow in the tangential direction to attain a better distribution without causing much penalty from the SD error term. Meanwhile, as desired, such a flow is dampened at a high-curvature region due to the role played by the curvature radius $\rho$ and distance $d$ in the SD error term. In contrast, tangential flow of control points is inhibited by the PD error term, causing stagnant improvement. Meanwhile, this tangential flow is checked nowhere by the TD error term since the TD error term ignores curvature variation on the fitting curve, thus leading to unstable convergence in the presence of corner points in the target shape. 
The ease of implementation and per iteration computation time of SDM are nearly the same as those of PDM and TDM since the three methods share the same framework but use different quadratic error terms. The per iteration computation time of SDM is mainly determined by the number of data points. The dominant part of computation time is the computation of the foot points of all data points in each iteration. For example, for the set of 1,630 data points used in Example 3, computation of each iteration takes about 0.15 seconds on a PC with Pentium IV 2.4GHz CPU and 256MB RAM with over 95\% of this time spent on foot point computation.

\section{IMPLEMENTATION ISSUES}

In this section, we discuss the following implementation issues for facilitating the convergence or improving the computational efficiency of SDM: 1) initialization and adjustment of control points; 2) fast setup of error terms; and 3) adapting SDM to fit an open B-spline curve to data points.

\subsection{Initialization and Adjustment of Control Points}

All the three methods we have discussed so far, PDM, TDM, and SDM, are local minimization schemes; that is to say, their convergence depends on the initial value, that is, the initial fitting curve. We would like to point out several possibilities of specification of the initial fitting curve though this is not a focus of the present article. The first obvious option is to let the user specify an initial B-spline fitting curve that is sufficiently close to the target shape and has an appropriate number of control points.

For a target shape defined by a set of dense points, an alternative is to compute a quadtree partition of the data points and then extract a sequence of points approximating the target shape from nonempty cells, that is, those cells containing at least one data point. These extracted points can then be used as the control points of an initial B-spline fitting curve. Our experience shows that this method tends to produce too many control points at the beginning so control point deletion is normally required during the fitting process in order to obtain a fitting curve with a minimal number of control points while still meeting a prescribed error threshold.

Another approach under our current investigation is based on the active contour model. In this method, a simple initial fitting curve is specified either automatically or by the user. Then some external energy/force is used in combination with SDM to drive the fitting curve to converge to a complex target shape in a manner that ensures global convergence. The key issues here are (i) proper design of the external force so that features and concavities of the target shape can be captured; and (ii) progressive insertion of control points at appropriate locations and stages so as to provide increasing shape flexibility to cope with the complexity of the target shape. The details of this procedure are beyond the scope of the present article and we wish to report on them in a separate article. We refer to Yang et al. [2004] for discussions on control point insertion and deletion in the context of a local B-spline curve fitting procedure.

\subsection{Fast Setup of Error Terms}

Efficient computation of foot points on the fitting curve of data points is important especially when there are a large number of data points since an error term needs to be computed for each data point in every iteration. We use the following speedup method consisting of two phases: preprocessing and query. In preprocessing, we first compute a uniform spatial partition of the data points with a proper cell size and record those nonempty cells. Next, we sample a sufficient number of points on the fitting curve and compute the normal lines of the fitting curve at these sample points. Then we record the intersections between these normal lines and all the nonempty cells.

In the query phase, for each data point $X_{k}$, we find its containing cell and the two normal lines such that $X_{k}$ is between the two lines and they are closest to $X_{k}$ (see Figure 11). Let the two normal lines be 


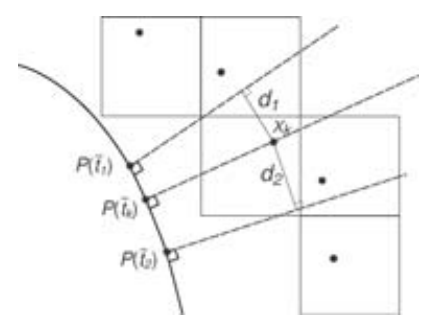

Fig. 11. Foot-point computation on a fitting curve.

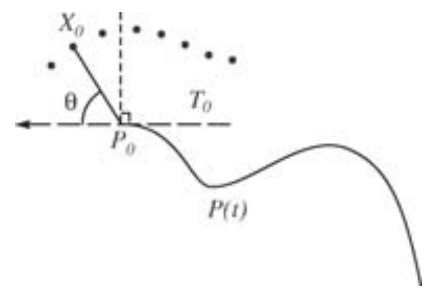

Fig. 12. Deriving an error term for an outer data point $X$.

associated with parameter values $\bar{t}_{1}$ and $\bar{t}_{2}$ of the fitting curve. Let $d_{1}$ and $d_{2}$ denote the distances from $X_{k}$ to the two lines. Then, supposing that the current fitting curve is sufficiently close to the target shape, a good estimate $P\left(\tilde{t}_{k}\right)$ of the foot-point of $X_{k}$ is given by linear interpolation $\tilde{t}_{k}=\left(d_{2} \bar{t}_{1}+d_{1} \bar{t}_{2}\right) /\left(d_{1}+d_{2}\right)$. The point $P\left(\tilde{t}_{k}\right)$ is then used as an initial point in a Newton-like iterative procedure to find the foot-point $P\left(t_{k}\right)$ of $X_{k}$.

Figure 13 shows an example of using SDM and PDM to fit a B-spline curve to the contour of a Chinese character Tian, meaning sky. In this example, the procedures described are used for initial fitting curve specification and control point insertion. Again we see that, to achieve the same level of fitting quality, PDM needs about the same number of control points but many more iterations than SDM does. TDM without step size control fails to converge for this example because the font outline has a number of high curvature feature points.

\subsection{Fitting an Open B-Spline Curve}

SDM can also be used to fit an open curve to a point cloud that represents an open target curve with some necessary modifications to ensure that the endpoints of the fitting curve are properly determined. We assume that the target curve is not self-intersecting and that a proper initial shape of an open fitting curve is provided. The data points near an end of the target curve are called target endpoints. There are two cases to consider.

Case 1: Some data points cannot be projected to inner points of the fitting curve; such points are called outer data points with respect to the fitting curve under consideration.

Case 2: All data points can be projected to inner points of the fitting curve.

In the first case, the error term associated with an outer data point is derived by blending the SD error term and the PD error term. Specifically, referring to Figure 12, let $T_{0}$ be the unit tangent vector of the fitting curve $P(t)$ at its endpoint $P_{0}$. Let $X_{0}$ be an outer point such that $P_{0}$ is the closest point from the curve $P(t)$ to $X_{0}$. Let $\theta$ denote the angle between the tangent line of the curve $P(t)$ at $P_{0}$ and the vector $X_{0}-P_{0}$, with $|\theta|<\pi / 2$ (since $X_{0}$ is an outer point). Then the error term $e_{\text {outer }, 0}$ to be used 


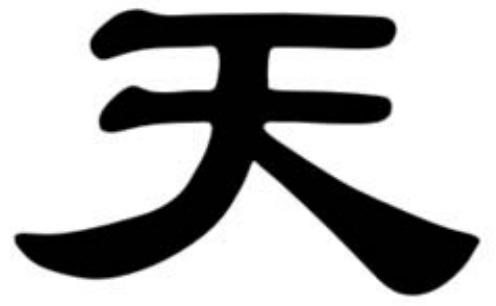

(a) A Chinese character, tian.

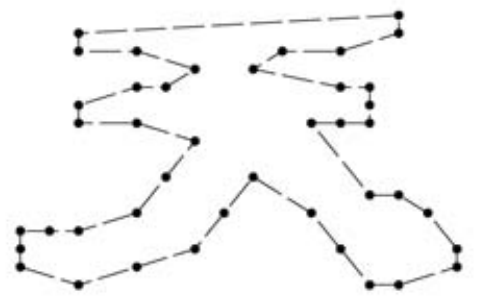

(c) 42 control points of an initial B-spline curve.

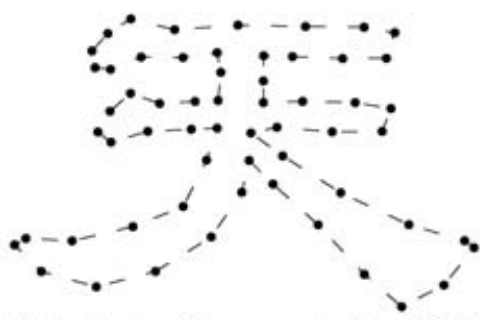

(e) Control points generated by SDM.

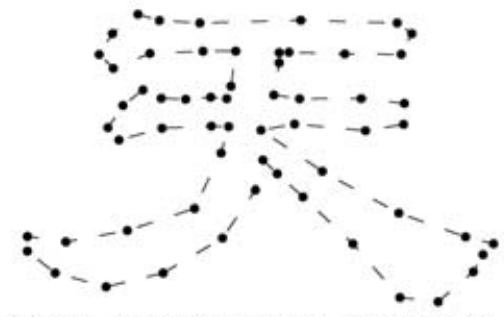

(g) Control points generated by PDM.

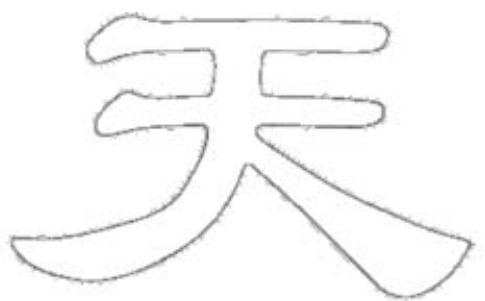

(b) The contour of the character (2,656 points).

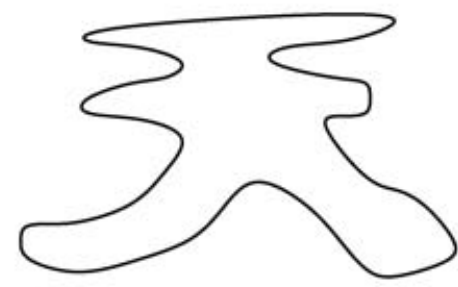

(d) The initial B-spline curve.

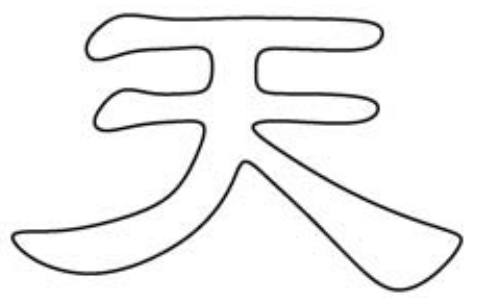

(f) The B-spline fitting curve from (e).

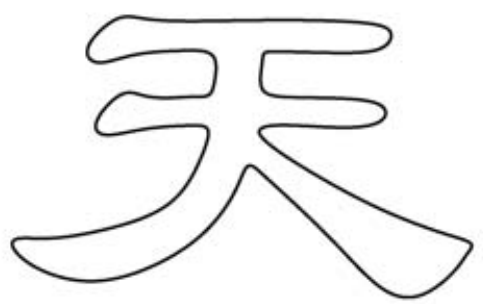

(h) The B-spline fitting curve from (g).

Fig. 13. SDM and PDM are applied to fitting a B-spline curve to the contour of a Chinese character in (a). The initial fitting curve in $(d)$ has the control points in (c) that are extracted from a quad-tree partition of the contour data points in (b). The coefficient of the smoothing term is $\lambda=0.005$. SDM produces the fitting curve in (f) with the 59 control points shown in (e) after 54 iterations, and PDM produces the fitting curve in (h) with the 60 control points in (g) after 352 iterations. 


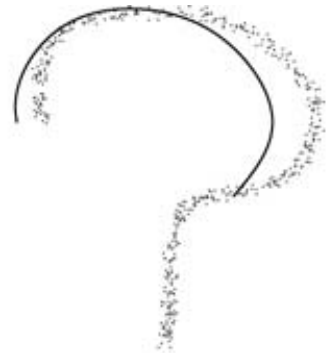

(a) An open target shape and an initial open B-spline curve with 8 control points.

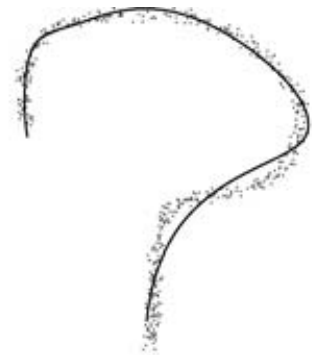

(b) The fitting curve generated by PDM in 20 iterations.

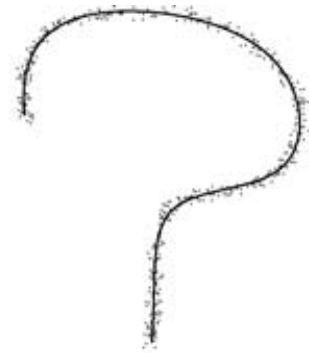

(c) The fitting curve generated by TDM in 20 iterations.

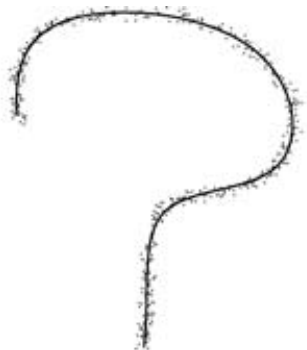

(d) The fitting curve generated by SDM in 20 iterations.

Fig. 14. Comparison of the three methods for fitting an open curve to a set of 472 data points. PDM needs about 600 iterations to reach the small approximation error of SDM as shown in (c).

for $X_{0}$ is given by the following interpolation of the PD error term and SD error term,

$$
e_{\text {outer }, 0}=\cos \theta e_{P D, 0}+(1-\cos \theta) e_{S D, 0} .
$$

Here $P_{0}$ is regarded as a function of the control points.

The rationale behind the interpolation in Equation (13) is to use the PD error term partially for outer data points so that through iterative optimization the endpoint $P_{0}$ of the fitting curve is pulled towards the target endpoints; of course, the SD error terms are still used for all other nonouter points. Note that the outer points in a target shape are identified relative to the current fitting curve; therefore we may have different data points as outer points in every iteration.

In the second case where none of the data points is an outer point, we just use the standard SDM method, that is, we use the SDM error term for each data point to make the fitting curve contract to fit the target shape. A nonzero but small value of $\alpha$ for the energy term $F_{1}$ in Equation (12) can be used to speed up the speed of contraction.

We are going to present two examples of fitting open B-spline curves to data points, using the technique previously described in combination with SDM and PDM. The first example is shown in Figure 14. We note that, in this example, PDM takes about 600 iterations to reach the same approximation error that is achieved by SDM in 20 iterations. This is again due to the strong tangential flow of B-spline control points that is accommodated by the SDM error term. We note that TDM works effectively for this example as well.

The second example, shown in Figure 15, is an application to reconstruct a revolution surface from a point cloud scanned in by a laser range scanner, following a method proposed in Pottmann and Wallner [2001]. The basic idea is as follows. First, the rotation axis of the revolution surface is estimated from the data points. Then this axis is used to rotate the input data points in 3D (Figure 15(a)) into data points lying on a 2D plane (Figure 15(b)) from which the B-spline profile curve is reconstructed using SDM. Then this profile curve is used to generate a revolution surface (Figure 15(c)) approximating the input 3D data points.

\section{DISCUSSION FROM THE VIEWPOINT OF OPTIMIZATION}

In this section, we discuss SDM, PDM, and TDM for B-spline curve approximation from the viewpoint of optimization. The B-spline curve fitting problem, as formulated in Equation (1), can also be seen as 


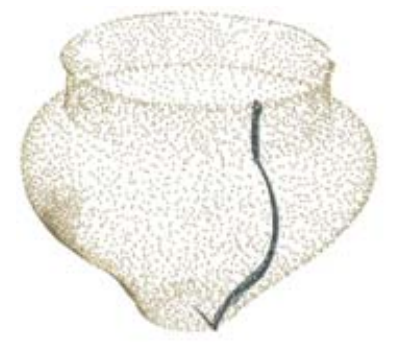

(a)

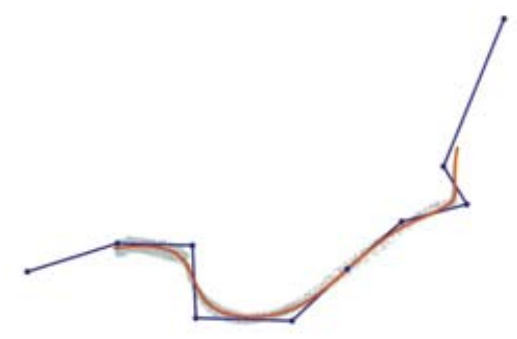

(b)

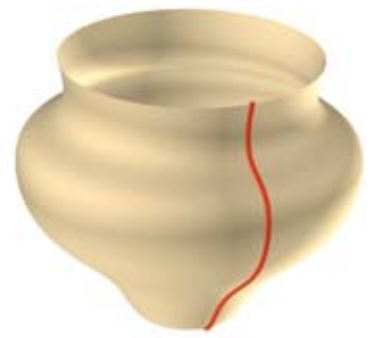

(c)

Fig. 15. Reconstruction of a revolution surface from a point cloud using a B-spline profile curve computed by SDM. (a) A revolution surface represented by a set of 5,077 points sampled from an original scan data of 423,697 points; (b) The thick planar point cloud is fitted with a uniform cubic B-spline curve computed by SDM; (c) The reconstructed revolution surface.

the nonlinear optimization problem of minimizing

$$
f=\frac{1}{2} \sum_{k=1}^{n}\left\|P\left(t_{k}\right)-X_{k}\right\|^{2}+\lambda f_{s}
$$

where $P\left(t_{k}\right)$ is a normal foot point of $X_{k}$, i.e.,

$$
\left(P\left(t_{k}\right)-X_{k}\right)^{T} P_{t}^{\prime}\left(t_{k}\right)=0, \quad k=1,2, \ldots, n .
$$

We note that, by treating $\mathcal{P}=\left\{P_{i}\right\}$ and $\mathcal{T}=\left\{t_{k}\right\}$ as two separable groups of variables and Equation (15) as constraints, this curve fitting problem is an instance of a separable and constrained nonlinear least squares problem for which the variable projection method using Gauss-Newton iteration often provides an efficient algorithm [Bjorck 1996]. This viewpoint will be helpful in the following computation of gradient and Hessian of the objective function $f$. For simplicity of discussion, in the following, we will ignore the regularization term $f_{s}$; our conclusion is still applicable with $f_{s}$ being taken into consideration since $f_{s}$ is independent of the $t_{k}$ and quadratic in the $P_{i}$, assuming that $\lambda$ is a fixed constant throughout all iterations.

In the rest of this section, we will first explain why PDM has linear convergence by viewing it as an alternating method. We will then investigate the standard algorithms for nonlinear least squares problems, namely Gauss-Newton iteration and the Levenberg-Marquart method [Kelley 1999], in connection with TDM. We will show that the Gauss-Newton method based on variable projection [Bjorck 1996] is exactly the same as TDM which is used in Blake and Isard [1998]. From this, we conclude on scenarios where TDM works well: a good initial position of the fitting curve and a small residual problem (i.e., data points are close to the final fitting curve); for a zero residual problem, optimization theory tells us that this method exhibits even quadratic convergence. The Levenberg-Marquart method is seen as a regularized version of TDM.

Our SDM scheme is finer than these standard methods (i.e., gradient descent and Gauss-Newton) for curve fitting as a nonlinear least squares problem. Although SDM is not a full Newton method because we do not use the complete Hessian, it comes close to it. In SDM, we approximate the complete Hessian by a quadratic approximation to the squared distance to a fitting curve in a manner that makes SDM adaptable to local curvature variation.

In fact, all of these methods can be seen as gradient descent schemes in some appropriate metric. While SDM chooses carefully the metric and TDM does this at least close to the target shape, PDM uses a metric which is not well adapted at all. Finally, we mention step size control [Kelley 1999] as a means of global convergence improvement of the three methods. 


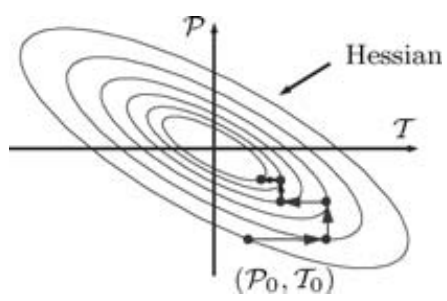

Fig. 16. The alternating minimization steps of PDM near a local minimum. $\mathcal{P}$ and $\mathcal{T}$ stand for the linear subspaces spanned by the control points and the data parameters, respectively.

\subsection{PDM as an Alternating Method}

We now explain why PDM is a variant of the steepest descent method and, therefore, has a linear convergence rate. Given a planar B-spline fitting curve $P(t)$ with control points $P_{i}$ and data points $X_{k}$, PDM minimizes the error function $f(\mathcal{P}, \mathcal{T})$ defined by Equation (14) which is a function in the Euclidean space $E^{2 m+n}$ spanned by $\mathcal{P}$ and $\mathcal{T}$, where $\mathcal{P}=\left\{P_{i}\right\}_{i=1}^{m}$ are the control points of the fitting curve and $\mathcal{T}=\left\{t_{k}\right\}_{k=1}^{n}$ are the parameter values associated the data points $X_{k}$.

PDM has the following two steps that are carried out in each iteration (see Figure 16): (1) for fixed parameter values $\mathcal{T}_{0}=\left\{t_{k, 0}\right\}$ and current control points $\mathcal{P}_{0}=\left\{P_{i, 0}\right\}$, find new control points $\mathcal{P}_{1}=\left\{P_{i, 1}\right\}$ by minimization of the quadratic function $f\left(\mathcal{P}, \mathcal{T}_{0}\right)$; $(2)$ considering the control points $\mathcal{P}_{1}$ produced in Step 1 as fixed, find new parameter values $\mathcal{T}_{1}=\left\{t_{k, 1}\right\}$ by minimization of the error function $f\left(\mathcal{P}_{1}, \mathcal{T}\right)$; this is done by computing the foot-points $P\left(t_{k}\right)$ of the data points $X_{k}$ on the fitting curve $P(t)$ with the control points $\mathcal{P}_{1}$. Thus, PDM minimizes the objective function in two subspaces parallel to $\mathcal{P}$ and $\mathcal{T}$, respectively, leading to a zigzag path near a local minimum as shown in Figure 16 which is reminiscent of the crawling behavior of the gradient descent method.

Due to the separate and alternate minimization of the $\mathcal{P}$ and $\mathcal{T}$ variables in each iteration, PDM is an alternating method which is a typical optimization technique for solving a separable nonlinear least squares problem and is known to have only linear convergence [Bjorck 1996].

\subsection{TDM-Gauss-Newton Iteration and Its Variants}

First we will derive an expression of the gradient of the objective function in Equation (14) which can also be regarded as a function of the $m$ control points $\mathcal{P}=\left(P_{1}, P_{2}, \ldots, P_{m}\right)$, that is, a function $f: R^{2 m} \rightarrow R$; the dependence of $\mathcal{T}$ on $\mathcal{P}$ is built through the constraints in Equation (15). For a fixed $k$, to indicate the dependence of $t_{k}$ on $\mathcal{P}$, we write $t_{k}=t(\mathcal{P})$, omitting the subscript for simplicity. Denote $\mathcal{F}=P\left(t_{k}\right)-X_{k}$, and $f_{k}=\|\mathcal{F}\|$. Then the constraints Equation (15) can be rewritten for each $k$ as

$$
\mathcal{F}_{t}^{T} \mathcal{F}=0 .
$$

Here and in the sequel, we will denote partial derivatives with a subscript, for example, $\mathcal{F}_{t}:=\partial \mathcal{F} / \partial t$, $\mathcal{F}_{t t}:=\partial^{2} \mathcal{F} / \partial t^{2}$.

We first compute the gradients of $f_{k}^{2}$ and $f_{k}$. Since $f_{k}^{2}=\mathcal{F}^{T} \mathcal{F}$, by Equation (16), we have

$$
\nabla f_{k}^{2}=\nabla\left(\mathcal{F}^{T} \mathcal{F}\right)=2\left(\mathcal{F}_{\mathcal{P}}^{T}+\nabla t \mathcal{F}_{t}^{T}\right) \mathcal{F}=2 \mathcal{F}_{\mathcal{P}}^{T} \mathcal{F}
$$

Here, $\nabla t$ is the gradient of $t_{k}$ with respect to $\mathcal{P}$, and $\mathcal{F}_{\mathcal{P}}$ is the matrix representing the partial derivative of $\mathcal{F}$ with respect to $\mathcal{P}$, not taking into account the dependency of $t_{k}$ on $\mathcal{P}$. Since $\nabla f_{k}^{2}=2 f_{k} \nabla f_{k}$, we obtain

$$
\nabla f_{k}=\mathcal{F}_{\mathcal{P}}^{T} \frac{\mathcal{F}}{f_{k}}=\mathcal{F}_{\mathcal{P}}^{T} \frac{\mathcal{F}}{\|\mathcal{F}\|}=-\mathcal{F}_{\mathcal{P}}^{T} N_{k}
$$


where $N_{k}=-\mathcal{F} /\|\mathcal{F}\|$ is a unit normal vector of the curve $P(t)$ at $P\left(t_{k}\right)$. Then the gradient of $f$ is found to be

$$
\nabla f=\frac{1}{2} \sum_{k} \nabla f_{k}^{2}=\left(\sum_{k=1}^{n} B_{1}\left(t_{k}\right)\left(P\left(t_{k}\right)-X_{k}\right), \ldots, \sum_{k=1}^{n} B_{m}\left(t_{k}\right)\left(P\left(t_{k}\right)-X_{k}\right)\right)^{T} .
$$

Each component of the above gradient vector, that is,

$$
(\nabla f)_{i}=\sum_{k=1}^{n} B_{i}\left(t_{k}\right)\left(P\left(t_{k}\right)-X_{k}\right),
$$

stands for a 2D vector associated with the $i$-th control point $P_{i}$, which is a weighted sum of the error vectors $P\left(t_{k}\right)-X_{k}$, where the weights are given by the $i$-th basis function $B_{i}$, evaluated at the parameter $t_{k}$ of the foot-point; of course, only those error vectors in the support of $B_{i}$ have influence.

At some places, it will be convenient to represent the B-spline curve in matrix form,

$$
P(t)=B(t) \mathcal{P},
$$

where $B(t)$ is the $2 \times 2 m$ matrix $\left(B_{1}(t) I_{2}, \ldots, B_{m}(t) I_{2}\right)$ with $I_{2}$ being the $2 \times 2$ identity matrix. Then the gradient vector $\nabla f \in R^{2 m}$ can be written as

$$
\nabla f=\sum_{k=1}^{n} B^{T}\left(t_{k}\right)\left(P\left(t_{k}\right)-X_{k}\right)=\sum_{k=1}^{n} B^{T}\left(t_{k}\right) B\left(t_{k}\right) \mathcal{P}-\sum_{k=1}^{n} B^{T}\left(t_{k}\right) X_{k} .
$$

Now let us consider the Gauss-Newton method. A Newton method minimizes the second-order approximant of the objective function at the current position $x_{c}$ to obtain the next iterate $x_{+}$. To find this quadratic approximant for a nonlinear least squares problem with $f=\frac{1}{2} \sum_{k} f_{k}^{2}$, one needs to compute the Hessian of $f$, which is

$$
\nabla^{2} f=\sum_{k=1}^{n} \nabla f_{k} \cdot\left(\nabla f_{k}\right)^{T}+\sum_{k=1}^{n} f_{k} \nabla^{2} f_{k}
$$

Since the computation of $\nabla^{2} f_{k}$ is usually too costly, the Gauss-Newton method uses only the first part in Equation (21) to approximate the Hessian $\nabla^{2} f$. This amounts to computing the minimizer $x_{+}$of the linear least squares problem

$$
\min \frac{1}{2} \sum_{k}\left[f_{k}\left(x_{c}\right)+\nabla\left(f_{k}\left(x_{c}\right)\right)^{T} \cdot\left(x-x_{c}\right)\right]^{2} .
$$

That is, a linear approximation of $f_{k}$ is used in the Gauss-Newton method.

In the B-spline curve fitting problem, the update step $x_{+}-x_{c}$ is given by the displacement vectors $\mathcal{D}=\left(D_{1}, \ldots, D_{m}\right)$ of the $m$ control points. From Equation (18) and Equation (19), since $\mathcal{F}=P\left(t_{k}\right)-X_{k}$, we have

$$
\nabla f_{k}=-\mathcal{F}_{\mathcal{P}}^{T} N_{k}=-B^{T}\left(t_{k}\right) N_{k} .
$$

Therefore, the Gauss-Newton iteration for B-spline curve fitting performs iterative minimization of

$$
f_{G N}=\frac{1}{2} \sum_{k}\left[f_{k}-\sum_{i} B_{i}\left(t_{k}\right) D_{i}^{T} N_{k}\right]^{2}
$$

which is interleaved with the step of foot-point computation in order to satisfy the constraints Equation (15). Since $N_{k}=\left(X_{k}-P\left(t_{k}\right)\right) /\left\|P\left(t_{k}\right)-X_{k}\right\|$, we have $f_{k}=\left(X_{k}-P\left(t_{k}\right)\right)^{T} N_{k}$. Noting that 
$P\left(t_{k}\right)=\sum_{i} B_{i}\left(t_{k}\right) P_{i}$, we obtain

$$
\begin{aligned}
f_{G N} & =\frac{1}{2} \sum_{k}\left[\left(X_{k}-P\left(t_{k}\right)\right)^{T} N_{k}-\sum_{i} B_{i}\left(t_{k}\right) D_{i}^{T} N_{k}\right]^{2}=\frac{1}{2} \sum_{k}\left[\left(X_{k}-\sum_{i} B_{i}\left(t_{k}\right)\left(P_{i}+D_{i}\right)\right)^{T} N_{k}\right]^{2} \\
& =\frac{1}{2} \sum_{k}\left[\left(X_{k}-P_{+}\left(t_{k}\right)\right)^{T} N_{k}\right]^{2}=\frac{1}{2} \sum_{k} e_{T D, k}
\end{aligned}
$$

where $e_{T D, k}$ is defined in Equation (4). Hence, the minimization of the TD error function in Equation (5) in TDM is equivalent to Gauss-Newton iteration.

The TD error term $e_{T D, k}$, unlike the SDM error term proposed in this article, counts for neither the distance from the data point $X_{k}$ to the curve $P(t)$ nor the curvature of the curve $P(t)$, reflecting the fact that the Gauss-Newton method omits the term $f_{k} \nabla^{2} f_{k}$ in Equation (21).

Strictly speaking, TDM is not the standard Gauss-Newton method, since there is a step of foot-point computation interleaved with Gauss-Newton iteration. In TDM, the Gauss-Newton step is applied in the tangent plane to the constraint surface defined by the constraints Equation (16). Such an algorithm is called a variable projection method using a Gauss-Newton method for solving a separable and constrained nonlinear least squares problem and can be shown [Ruhe and Wedin 1980; Bjorck 1996] to have the same asymptotic convergence behavior as the full Gauss-Newton method applied to all variables (i.e., $\mathcal{P}$ and $\mathcal{T}$ in our case).

TDM also shares the same framework of the so called generalized reduced gradient $(G R G)$ method [Luenberger 1984] for solving a nonlinear constraint problem with two separable groups of variables; the steepest gradient direction is used in the tangent plane of the constraint surface in the GRG method, while a Gauss-Newton step in used in TDM.

It is well known [Kelley 1999, p. 24] that, in a Gauss-Newton method, if $x_{c}$ is sufficiently close to the minimizer $x^{*}$ of $f$, the distance $\left\|e_{c}\right\|=\left\|x_{c}-x^{*}\right\|$ of the current iterate to $x^{*}$ is related to the error $\left\|e_{+}\right\|$ in the next iterate by

$$
\left\|e_{+}\right\| \leq K\left(\left\|e_{c}\right\|^{2}+\left\|R\left(x^{*}\right)\right\|\left\|e_{c}\right\|\right)
$$

where $R\left(x^{*}\right)=\left(f_{1}, \ldots, f_{n}\right)\left(x^{*}\right)$ is the residual at $x^{*}$, and $K$ is a constant which involves the Jacobian of $R(x)$. It follows from Equation (23) that, for a zero residual problem, Gauss-Newton iteration converges quadratically and the data points can be fitted exactly. Furthermore, Gauss-Newton iteration has fast convergence for good initial data and a small residual problem. For a large residual problem, the GaussNewton iteration may not converge at all.

Some variants of the Gauss-Newton method are possible. If only a scalar multiple of the GaussNewton step, $s\left(x_{+}-x_{c}\right)$, usually with $0<s<1$, is used for stepping to the next solution, then one obtains the damped Gauss-Newton method [Kelley 1999].

Another way to modify Gauss-Newton is a regularization with the Levenberg-Marquart method [Kelley 1999], in which a scalar multiple of the identity matrix is added to the approximate Hessian. In our setting, this method requires the minimization of

$$
f_{L M}=\frac{1}{2} \sum_{k}\left[\left(X_{k}-P_{+}\left(t_{k}\right)\right)^{T} N_{k}\right]^{2}+v_{c} \sum_{i}\left\|D_{i}\right\|^{2} .
$$

Thus, the regularization term penalizes large changes $D_{i}$ in the control points. It can be shown that using a regularization parameter $v_{c}$ of the order of the norm of the residual, that is, $O\left(\left\|R\left(x_{c}\right)\right\|\right)$, one still obtains quadratic convergence for a zero residual problem. A drawback of the Levenberg-Marquart method in the setting of curve fitting is that the same magnitude of regularization is applied to every control point without taking into account the curvature variation at different locations. 
By writing the fitting error as a function of the parameter values $t_{k}$, the Levenberg-Marquart method is used in Sarkar and Menq [1991] to iteratively update the $t_{k}$, and faster convergence of this method than a variant of PDM is reported. However, although the foot-point computation is avoided, it is noted in Sarkar and Menq [1991] that this variant of the L-M method is about 10 times slower than PDM per iteration.

A global Gauss-Newton method is implemented in Speer et al. [1998] to update the control points $P_{i}$ and the parameter values $t_{k}$ together, avoiding the costly step of computing foot-points of data points. However, a relatively large linear system of equations needs to be solved since now the parameter values of a large number of data points also enter optimization. The comparison of TDM with this global Gauss-Newton method as well as other variants of the L-M method is an interesting problem but beyond the scope of the present article.

\subsection{SDM-A Quasi-Newton Method}

In this section, we will derive the expression of the Newton method and then reveal the difference between our SDM scheme and the Newton method to show that SDM is, in fact, a quasi-Newton method. The key to this analysis is deriving a suitable expression of the Hessian of the objective function.

For a fixed $k$, consider the term $f_{k}^{2}=\mathcal{F}^{T} \mathcal{F}$. Denote $\mathcal{F}=\left(\mathcal{F}_{x}, \mathcal{F}_{y}\right)^{T}$. By Equation (17), we have $\nabla f_{k}^{2}=2 \mathcal{F}_{\mathcal{P}}^{T} \mathcal{F}$. The derivative of $\nabla f_{k}^{2}$ yields the Hessian

$$
\begin{aligned}
\frac{1}{2} \nabla^{2} f_{k}^{2}= & \mathcal{F}_{\mathcal{P}}^{T} \mathcal{F}_{\mathcal{P}}+\left(\mathcal{F}_{\mathcal{P}}^{T} \mathcal{F}_{t}+\mathcal{F}_{\mathcal{P} t}^{T} \mathcal{F}\right) \nabla t^{T}+\nabla t\left(\mathcal{F}_{t}^{T} \mathcal{F}_{\mathcal{P}}+\mathcal{F}^{T} \mathcal{F}_{\mathcal{P} t}\right)+\left(\mathcal{F}_{t t}^{T} \mathcal{F}+\mathcal{F}_{t}^{T} \mathcal{F}_{t}\right) \nabla t \nabla t^{T} \\
& +\mathcal{F}_{x} \mathcal{F}_{x \mathcal{P} P}+\mathcal{F}_{y} \mathcal{F}_{y \mathcal{P} P} \\
= & \mathcal{F}_{\mathcal{P}}^{T} \mathcal{F}_{\mathcal{P}}+\left(\mathcal{F}_{\mathcal{P}}^{T} \mathcal{F}_{t}+\mathcal{F}_{\mathcal{P} t}^{T} \mathcal{F}\right) \nabla t^{T}+\nabla t\left(\mathcal{F}_{t}^{T} \mathcal{F}_{\mathcal{P}}+\mathcal{F}^{T} \mathcal{F}_{\mathcal{P} t}\right)+\left(\mathcal{F}_{t t}^{T} \mathcal{F}+\mathcal{F}_{t}^{T} \mathcal{F}_{t}\right) \nabla t \nabla t^{T}
\end{aligned}
$$

Here we used the fact that $\mathcal{F}_{x \mathcal{P P}}=0, \mathcal{F}_{y \mathcal{P P}}=0$, since $\mathcal{F}$ is linear in $\mathcal{P}$. Again, $\nabla t$ stands for the gradient of $t_{k}=t(\mathcal{P})$ with respect to $\mathcal{P}$.

On the other hand, we need to find the relationship between $\nabla t$ and $\mathcal{F}_{\mathcal{P}}$. Differentiating the constraint Equation (16), we obtain

$$
\left(\mathcal{F}_{\mathcal{P} t}^{T}+\nabla t \mathcal{F}_{t t}^{T}\right) \mathcal{F}+\left(\mathcal{F}_{\mathcal{P}}^{T}+\nabla t \mathcal{F}_{t}^{T}\right) \mathcal{F}_{t}=0
$$

Solving for $\nabla t$ yields

$$
\nabla t=-\frac{\mathcal{F}_{\mathcal{P} t}^{T} \mathcal{F}+\mathcal{F}_{\mathcal{P}}^{T} \mathcal{F}_{t}}{\mathcal{F}_{t t}^{T} \mathcal{F}+\mathcal{F}_{t}^{T} \mathcal{F}_{t}}
$$

Substituting this expression of $\nabla t$ in Equation (24), we obtain the complete Hessian

$$
\frac{1}{2} \nabla^{2} f_{k}^{2}=\mathcal{F}_{\mathcal{P}}^{T} \mathcal{F}_{\mathcal{P}}-\frac{\left(\mathcal{F}_{\mathcal{P} t}^{T} \mathcal{F}+\mathcal{F}_{\mathcal{P}}^{T} \mathcal{F}_{t}\right)\left(\mathcal{F}_{t}^{T} \mathcal{F}_{\mathcal{P}}+\mathcal{F}^{T} \mathcal{F}_{\mathcal{P} t}\right)}{\mathcal{F}_{t t}^{T} \mathcal{F}+\mathcal{F}_{t}^{T} \mathcal{F}_{t}}
$$

We will now make a simplification by neglecting the term $\mathcal{F}_{\mathcal{P} t}^{T} \mathcal{F}$, that is, setting it to zero. This results in an approximate Hessian $\widetilde{\nabla}^{2} f_{k}^{2}$. To interpret this approximate Hessian geometrically, we introduce the arc length parameter $s$ of the B-spline curve $P(t)$. Then we have

$$
\begin{aligned}
\frac{1}{2} \widetilde{\nabla}^{2} f_{k}^{2} & =\mathcal{F}_{\mathcal{P}}^{T} \mathcal{F}_{\mathcal{P}}-\frac{\mathcal{F}_{\mathcal{P}}^{T} \mathcal{F}_{t} \mathcal{F}_{t}^{T} \mathcal{F}_{\mathcal{P}}}{\mathcal{F}_{t t}^{T} \mathcal{F}+\mathcal{F}_{t}^{T} \mathcal{F}_{t}}=\mathcal{F}_{\mathcal{P}}^{T} \mathcal{F}_{\mathcal{P}}-\frac{\left(s_{t}\right)^{2} \mathcal{F}_{\mathcal{P}}^{T} \mathcal{F}_{s} \mathcal{F}_{s}^{T} \mathcal{F}_{\mathcal{P}}}{\left[\mathcal{F}_{s s}^{T}\left(s_{t}\right)^{2}+\mathcal{F}_{s}^{T} s_{t t}\right] \mathcal{F}+\mathcal{F}_{s}^{T} \mathcal{F}_{s}\left(s_{t}\right)^{2}} \\
& =\mathcal{F}_{\mathcal{P}}^{T} \mathcal{F}_{\mathcal{P}}-\frac{\left(s_{t}\right)^{2} \mathcal{F}_{\mathcal{P}}^{T} \mathcal{F}_{s} \mathcal{F}_{s}^{T} \mathcal{F}_{\mathcal{P}}}{\mathcal{F}_{s s}^{T} \mathcal{F}\left(s_{t}\right)^{2}+\mathcal{F}_{s}^{T} \mathcal{F}_{s}\left(s_{t}\right)^{2}}=\mathcal{F}_{\mathcal{P}}^{T} \mathcal{F}_{\mathcal{P}}-\frac{\mathcal{F}_{\mathcal{P}}^{T} \mathcal{F}_{s} \mathcal{F}_{s}^{T} \mathcal{F}_{\mathcal{P}}}{\left(\mathcal{F}_{s s}^{T} \mathcal{F}+\mathcal{F}_{s}^{T} \mathcal{F}_{s}\right)}
\end{aligned}
$$

The term $\mathcal{F}_{s}^{T} \mathcal{F} s_{t t}$ drops out due to the constraint Equation (16) and the fact that $\mathcal{F}_{s}^{T}$ and $\mathcal{F}_{t}^{T}$ are collinear. ACM Transactions on Graphics, Vol. 25, No. 2, April 2006. 
Clearly, $\mathcal{F}_{s}^{T} \mathcal{F}_{s}=1$ since $\mathcal{F}_{s}=T_{k}$ is the unit tangent vector of $P(t)$. Since $\mathcal{F}_{s s}$ is the curvature vector of $P(t)$ at $P\left(t_{k}\right)$, we have $\mathcal{F}_{s s}^{T} \mathcal{F}=-d \kappa$, where $\kappa \geq 0$ is the curvature, and $d$ is the signed distance defined in Section 3. Hence, we obtain

$$
\frac{1}{2} \widetilde{\nabla}^{2} f_{k}^{2}=\mathcal{F}_{\mathcal{P}}^{T} \mathcal{F}_{\mathcal{P}}-\frac{\mathcal{F}_{\mathcal{P}}^{T} T_{k} T_{k}^{T} \mathcal{F}_{\mathcal{P}}}{-d \kappa+1}
$$

Noting that $T_{k} T_{k}^{T}+N_{k} N_{k}^{T}=I$, this equation is further rewritten as

$$
\frac{1}{2} \widetilde{\nabla}^{2} f_{k}^{2}=\mathcal{F}_{\mathcal{P}}^{T}\left(I-T_{k} T_{k}^{T}\right) \mathcal{F}_{\mathcal{P}}-\frac{d \kappa \mathcal{F}_{\mathcal{P}}^{T} T_{k} T_{k}^{T} \mathcal{F}_{\mathcal{P}}}{-d \kappa+1}=\mathcal{F}_{\mathcal{P}}^{T} N_{k} N_{k}^{T} \mathcal{F}_{\mathcal{P}}+\frac{d}{d-\rho} \mathcal{F}_{\mathcal{P}}^{T} T_{k} T_{k}^{T} \mathcal{F}_{\mathcal{P}}
$$

Now we consider the relationship between SDM and the quasi-Newton method obtained by replacing the Hessian $\nabla^{2} f_{k}^{2}$ by $\widetilde{\nabla}^{2} f_{k}^{2}$. Note that

$$
\mathcal{F}_{\mathcal{P}}^{T} N_{k} N_{k}^{T} \mathcal{F}_{\mathcal{P}}=\nabla f_{k}\left(\nabla f_{k}\right)^{T},
$$

which is the first term in Equation (21) that is used by Gauss-Newton iteration to approximate the true Hessian. Thus, replacing the Hessian $\nabla^{2} f_{k}^{2}$ by $\widetilde{\nabla}^{2} f_{k}^{2}$ in the Newton method is equivalent to adding the second term in Equation (27) to the Gauss-Newton method to yield a quasi-Newton method. Recall that the Gauss-Newton method is the same as TDM. Therefore, noting that $\left(P\left(t_{k}\right)-X_{k}\right)^{T} T_{k}=0$ (by Equation (15)), the quasi-Newton method minimizes the quadratic function

$$
\begin{aligned}
f_{Q N} & =f_{G N}+\frac{1}{2} \sum_{k=1}^{n} \mathcal{D}\left(\frac{d_{k}}{d_{k}-\rho_{k}} \mathcal{F}_{\mathcal{P}}^{T} T_{k} T_{k}^{T} \mathcal{F}_{\mathcal{P}}\right) \mathcal{D}^{T} \\
& =f_{G N}+\frac{1}{2} \sum_{k=1}^{n} \frac{d_{k}}{d_{k}-\rho_{k}}\left[\left(\sum_{i} B_{i}\left(t_{k}\right) D_{i}\right)^{T} T_{k}\right]^{2} \\
& =\frac{1}{2} \sum_{k=1}^{n}\left\{\left[\left(P_{+}\left(t_{k}\right)-X_{k}\right)^{T} N_{k}\right]^{2}+\frac{d_{k}}{d_{k}-\rho_{k}}\left[\left(P_{+}\left(t_{k}\right)-P\left(t_{k}\right)\right)^{T} T_{k}\right]^{2}\right\} \\
& =\frac{1}{2} \sum_{k=1}^{n}\left\{\left[\left(P_{+}\left(t_{k}\right)-X_{k}\right)^{T} N_{k}\right]^{2}+\frac{d_{k}}{d_{k}-\rho_{k}}\left[\left(P_{+}\left(t_{k}\right)-X_{k}\right)^{T} T_{k}\right]^{2}\right\}=\frac{1}{2} \sum_{k=1}^{n} h_{k}(\mathcal{D}),
\end{aligned}
$$

where $h_{k}(\mathcal{D})$ is defined in Equation (9), and $d_{k}, \rho_{k}$ are the corresponding distance and curvature radius for the $k$-th term. Hence, the quasi-Newton method uses a local quadratic model $f_{Q N}$ that is the same as the quadratic approximant $h_{k}(\mathcal{D})$ used by the SD error term before it is turned into the semidefinite form in Equation (10). Hence, we have shown that the SDM method is a quasi-Newton method obtained by discarding the term $\mathcal{F}^{T} \mathcal{F}_{\mathcal{P} t}$ which amounts to disregarding the change $\mathcal{F}_{\mathcal{P} t}$ of the tangent vector $P_{t}^{\prime}\left(t_{k}\right)$ caused by the change of the control points.

SDM does not fall into the category of the quasi-Newton methods that fulfills the so-called secant equation [Kelley 1999]. Instead, SDM uses another positive definite approximant of the Hessian based on geometric considerations. Although SDM is not a standard optimization procedure, it is a computationally attractive and effective compromise between a full Newton scheme and Gauss-Newton; it picks up more contributions of the true Hessian than the Gauss-Newton iteration (or TDM) does but ignores the remaining part for reasons of computational efficiency and simplicity. Indeed, SDM is an optimization scheme that is particularly suited for solving shape fitting problems because it uses an intuitively simple error metric that is adaptable to local curvature variation of a target shape.

Each term $f_{k}^{2}$ in the objective function in Equation (14) is the squared distance function. Because the exact Hessian in the second order Taylor expansion of $f_{k}^{2}$ is modified in order to derive the SD 


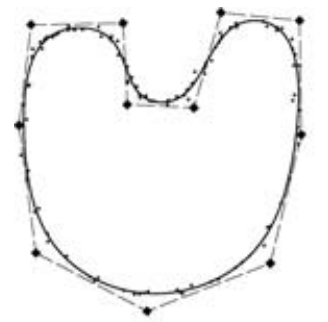

(a)

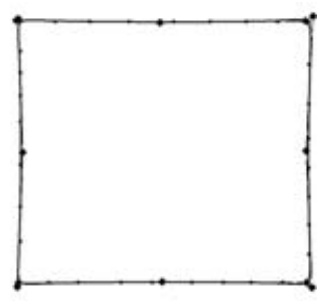

(b)

Fig. 17. TDM using the Armijo rule for step size control. (a) The fitting curve by TDM in 20 iterations for the data set in Figure 7(a); (b) The fitting curve by TDM in 20 iterations for the data set in Figure 10(a).

error term, we conclude that, in general, the SD error term is not a second order approximation to the squared distance even without the modification in Equation (10) to make the error term positive semidefinite. This is in contrast to the previous squared distance minimization method by Pottmann et al. [2002] where the quadratic approximant in Equation (6) is always a second order approximation to the squared distance function before the modification to turn it into the positive semidefinite error term in Equation (7).

\subsection{Step Size Control}

We have tested step size control on PDM, TDM, and SDM, using the Armijo rule [Kelley 1999]. It is found that step size control does not help much with PDM and SDM-PDM still converges slowly, and the periteration computation of SDM becomes much longer with a moderate degree of improvement in stability. The stability of TDM improves greatly with the help of step size control. However, at the cost of much longer per iteration time especially when approaching a local minimum since, due to the flat gradient near a local minimum, it normally gets more time consuming to select an appropriate step size via repetitive evaluations of the fitting error.

Figure 17 shows the result of applying step size control (the Armijo rule) to TDM on the same data points and initial B-spline curves as shown in Figures 7(a) and 10(a); now both data sets are satisfactorily approximated by B-spline curves computed with TDM. For comparison, refer to Figures 7(c) and 10(c) to see the unacceptable fitting curves generated by TDM without step size control.

\section{CONCLUDING REMARKS}

PDM is widely used in practice for parametric curve and surface fitting [Farin 1997]. As we have shown, PDM has linear convergence in theory, converges slowly in practice, and is often trapped in a poor local minimum. TDM is a method often used for curve fitting in computer vision (e.g., Blake and Isard [1998]). TDM converges faster than PDM but its convergence is highly unstable. Against this backdrop, we have proposed a novel and efficient method, called SDM, for fitting B-spline curves to point cloud data. We have shown empirically that SDM converges faster than PDM and that SDM is more stable than TDM. In addition, SDM is easy to implement and has similar per iteration computation time to PDM and TDM since they share the same framework. All this suggests that SDM is a favorable alternative to PDM or TDM for B-spline curve fitting.

In order to gain a better understanding of the above geometrically motivated schemes, we have studied the B-spline curve fitting problem from the optimization viewpoint. We note that PDM is a gradient descent method in a metric that is not well chosen. We have also shown that TDM uses a Gauss-Newton step for solving a nonlinear least squares problem, and its instability at high curvature regions is thus due to its omission of important parts in the true Hessian of the objective function and the lack of 
step size control. Finally, we have shown that our proposed SDM scheme is a quasi-Newton method using a carefully chosen approximate Hessian, and thus its superior performance in both convergence and stability does not come as a surprise. Interestingly, unlike most other quasi-Newton methods, the approximate Hessian used by SDM is not explicitly computed; it arises naturally as the consequence of using the simple SDM error term devised out of entirely geometric considerations, that is, making use of curvature information to give a close approximation of the squared distance function. This contributes to the simplicity and efficiency of SDM.

We expect to see more studies on SDM and related problems both theoretically and from the viewpoint of applications. An immediate extension is to apply SDM to optimize the weights and knots of a NURBS fitting curve, an issued discussed in Laurent-Gengoux and Mekhilef [1993] and Goldenthal and Bercovier [2004]. Other significant problems include the analysis of the convergence rate of SDM and the improvement of the global convergence to the target shape from a very simple seed shape; the latter topic has a close connection to the work on active contours [Kass et al. 1988; Malladi et al. 1995; Osher and Fedkiw 2003; Sethian 1999].

Our ongoing research shows that SDM can be applied to a large class of shape reconstruction and geometric optimization problems such as fitting B-spline surfaces or subdivision surfaces, optimization over an analytical surface or a mesh surface, and surface registration. This extension of SDM to the surface case would be of great practical importance in view of the current use of PDM as a predominant optimization method for surface fitting in graphics and CAD.

\section{REFERENCES}

Ambrosio, L. and Montegazza, C. 1998 . Curvature and distance function from a manifold. J. Geomet. Analy. 8, 723-748.

Bercovier, M. and Jacob, M. 1994. Minimization, constraints and composite bézier curves. Comput. Aided Geomet. Design 11, $533-563$.

BJorck, A. 1996. Numerical Methods for Least Squares Problems. Mathematics Society for Industrial and Applied Mathematics, Philadelphia, PA.

Blake, A. And Isard, M. 1998. Active Contours. Springer Verlag, New York, NY.

DJEbali, M., MElKemi, M., AND SAPIDIS, N. 2002. Range-image segmentation and model reconstruction based on a fit-and-merge strategy. In Proceedings of the 7th ACM Symposium on Solid Modeling and Applications. 127-138.

FARIN, G. 1997. Curves and Surfaces for Computer Aided Geometric Design: A Practical Guide, 4th Ed. Academic Press, New York, NY.

Forsey, D. R. AND Bartels, R. H. 1995. Surface fitting with hierarchical splines. ACM Trans. Graph. 14, $134-161$.

Goldenthal, R. and Bercovier, M. 2004. Spline curve approximation and design by optimal control over the knots. Computing 72, 53-64.

Goshtasby, A. A. 2000. Grouping and parameterizing irregularly spaced points for curve fitting. ACM Trans. Graph. 19, 185-203.

Greiner, G., Kolb, A., ANd RiePl, A. 2002. Scattered data interpolation using data dependant optimization techniques. Graphical Models 64, 1-18.

Haber, J., Zeilfelder, F., Davydov, O., ANd Seidel, H. P. 2001. Smooth approximation and rendering of large scattered data sets. In Proceedings of Visualization. 341-348.

Hoppe, H. 1996. Progressive meshes. In Proceedings of SIGGRAPH. 99-108.

Hoppe, H., DeRose, T., Duchamp, T., Halstead, M., Jin, H., McDonald, J., Schweitzer, J., and Stuetzle, W. $1994 . \quad$ Piecewise smooth surface reconstruction. In Proceedings of SIGGRAPH. 295-302.

Ноsснек, J. 1988. Intrinsic parameterization for approximation. Comput. Aided Geomet. Design 5, 27-31.

Hoschek, J. And Lasser, D. 1993. Fundamentals of Computer Aided Geometric Design. AK Peters.

Hu, S. M. AND Wallner, J. 2005. Second order algorithm for orthogonal projection onto curves and surfaces. Comput. Aided Geomet. Design 22, 251-260.

Kass, M., Witkin, A., And Terzopoulos, D. 1988. Snakes: Active contour models. Int. J. Comput. Vision 1, 321-331.

Kelley, C. T. 1999. Iterative Methods for Optimization. Society for Industrial and Applied Mathematics, Philadelphia, PA.

Laurent-Gengoux, P. and Mekhilef, M. 1993. Optimization of a nurbs representation. Comput.-aided Design 25, 699-710. 
LeE, E. T. Y. 1989. Choosing nodes in parametric curve interpolation. Comput. -aided Design 21, 363-370.

Luenberger, D. 1984. Linear and Nonlinear Programming. Addision-Wesley.

MA, W. Y. AND KRUTH, J. P. 1995. Parameterization of randomly measured points for least squares fitting of b-spline curves and surfaces. Comput.-aided Design 27, 663-675.

MaekaWA, I. ANd Ko, K. 2002. Surface construction by fitting unorganized curves. Graphical Models 64, 316-332.

Malladi, R., Sethian, J., and Vemuri, B. C. 1995. Shape modeling with front propagation: A level set approach. IEEE Trans. Pattern Analy. Mach. Intell. 17, 158-175.

Osher, S. And Fedkiw. 2003. Level Set Methods and Dynamic Implicit Surfaces. Springer-Verlag, New York, NY.

PAvlidis, T. 1983. Curve fitting with conic splines. ACM Trans. Graph. 2, 1-31.

Plass, M. and Stone, M. 1983. Curve-fitting with piecewise parametric cubics. Comput. Graphics 17, 3, $229-239$.

Pottmann, H. and Hofer, M. 2003. Geometry of the squared distance function to curves and surfaces. In Visualization and Mathematics III, H. Hege and K. Polthier, Eds. 223-244.

Pottmann, H., Leopoldseder, S., ANd Hofer, M. 2002. Approximation with active b-spline curves and surfaces. In Proceedings of Pacific Graphics. IEEE Computer Society Press, 8-25.

Pottmann, H. and Wallner, J. 2001. Computational Line Geometry. Springer-Verlag, Berlin, Germany.

PratT, V. 1985. Techniques for conic splines. In Proceedings of SIGGRAPH. 151-160.

Ruhe, A. ANd WEdin, P. 1980. Algorithms for separable nonlinear least squares problems. SIAM Rev. 22, $318-337$.

Sarkar, B. And Menq, C.-H. 1991. Parameter optimization in approximating curves and surfaces to measurement data. Comput. Aided Geomet. Design 8, 267-290.

Saux, E. ANd Daniel, M. 2003. An improved hoschek intrinsic parametrization. Comput. Aided Geomet. Design $20,513-521$. Sethian, J. A. 1999. Level Set Methods and Fast Marching Methods. Cambridge University Press, Cambridge, UK.

Speer, T., Kuppe, M., And Hoschek, J. 1998. Global reparameterization for curve approximation. Comput. Aided Geomet. Design 15, 869-877.

Taubin, G. 2002. Dual mesh resampling. Graphical Models 64, 94-113.

Walton, D. J. AND Xu, R. 1991. Turning point preserving planar interpolation. ACM Trans. Graph. 10, $297-311$.

Wang, X. C. And Phillips, C. 2002. Multi-weight enveloping: least-squares approximation techniques for skin animation. In Proceedings of the ACM SIGGRAPH / Eurographics Symposium on Computer Animation. 129-138.

Weiss, V., Andor, L., Renner, G., And Varady, T. 2002. Advanced surface fitting techniques. Comput.-Aided Geomet. Design 19, $19-42$.

YANG, H. P., WANG, W., AND Sun, J. G. 2004. Control point adjustment for b-spline curve approximation. Comput.-aided Design 36, 639-652.

Received August 2004; revised August 2005; accepted October 2005 